\title{
A practical guide to computer simulations *
}

\author{
Alexander K. Hartmann \\ University of Göttingen, Germany \\ hartmann@theorie.physik.uni-goettingen.de \\ Heiko Rieger \\ University of Saarbrücken, Germany \\ rieger@lusi.uni-sb.de
}

February 1, 2008

\begin{abstract}
Here practical aspects of conducting research via computer simulations are discussed. The following issues are addressed: software engineering, object-oriented software development, programming style, macros, make files, scripts, libraries, random numbers, testing, debugging, data plotting, curve fitting, finite-size scaling, information retrieval, and preparing presentations.

Because of the limited space, usually only short introductions to the specific areas are given and references to more extensive literature are cited. All examples of code are in $\mathrm{C} / \mathrm{C}++$.
\end{abstract}

\section{Contents}

1 Software Engineering 3

2 Object-oriented Software Development 10

3 Programming Style 16

4 Programming Tools 20

4.1 Using Macros . . . . . . . . . . . . . . . . . . 20

4.2 Make Files . . . . . . . . . . . . . . . . . . . 24

4.3 Scripts . . . . . . . . . . . . . . . . . . . 28

${ }^{*}$ Taken from the book: A.K. Hartmann and H. Rieger, Optimization Algorithms in Physics, (Wiley-VCH, Berlin, Weinheim 2001), ISBN 3-527-40307-8, with permission of Wiley-VCH, see http://www.wiley.com. This document may be distributed freely in electronic and nonelectronic form, provided that no changes are performed to it. 
5 Libraries 29

5.1 Numerical Recipes . . . . . . . . . . . . . . . . . . . . . 29

5.2 LEDA . . . . . . . . . . . . . . . . . . . . 31

5.3 Creating your own Libraries . . . . . . . . . . . . . . . 33

6 Random Numbers $\quad 34$

6.1 Generating Random Numbers . . . . . . . . . . . . . . . . . 35

6.2 Inversion Method . . . . . . . . . . . . . . . . . . . . 38

6.3 Rejection Method . . . . . . . . . . . . . . . . . . 40

6.4 The Gaussian Distribution . . . . . . . . . . . . . . . . . 41

7 Tools for Testing $\quad 42$

$7.1 \quad g d b \ldots \ldots \ldots \ldots \ldots \ldots \ldots \ldots \ldots \ldots$

$7.2 d d d \ldots \ldots \ldots \ldots \ldots \ldots \ldots \ldots \ldots \ldots$

$7.3 \quad$ checkergcc . . . . . . . . . . . . . . . 45

8 Evaluating Data 49

8.1 Data Plotting . . . . . . . . . . . . . . . . . . 49

8.2 Curve Fitting . . . . . . . . . . . . . . . . . . . 51

8.3 Finite-size Scaling . . . . . . . . . . . . . . . 55

9 Information Retrieval and Publishing $\quad 57$

9.1 Searching for Literature . . . . . . . . . . . . . . . 58

9.2 Preparing Publications . . . . . . . . . . . . . . . 61

Here practical aspects of conducting research via computer simulations are discussed. It is assumed that you are familiar with an operating system such as UNIX (e.g. Linux), a high-level programming language such as C, Fortran or Pascal and have some experience with at least small software projects.

Because of the limited space, usually only short introductions to the specific areas are given and references to more extensive literature are cited. All examples of code are in $\mathrm{C} / \mathrm{C}++$.

First, a short introduction to software engineering is given and several hints allowing the construction of efficient and reliable code are stated. In the second section a short introduction to object-oriented software development is presented. In particular, it is shown that this kind of programming style can be achieved with standard procedural languages such as C as well. Next, practical hints concerning the actual process of writing the code are given. In the fourth section macros are introduced. Then it is shown how the development of larger pieces of code can be organized with the help of so called make files. In the subsequent section the benefit of using libraries like Numerical Recipes or LEDA are explained and it is shown how you can build your own libraries. In the sixth section the generation of random numbers is covered while in the eighth section three very useful debugging tools are presented. Afterwards, programs to perform data analysis, curve fitting and finite-size scaling are explained. In the last section an introduction to information retrieval and literature search in the Internet and to the preparation of presentations and publications is given. 


\section{Software Engineering}

When you are creating a program, you should never just start writing the code. In this way only tiny software projects such as scripts can be completed successfully. Otherwise your code will probably be very inflexible and contain several hidden errors which are very hard to find. If several people are involved in a project, it is obvious that a considerable amount of planning is necessary.

But even when you are programming alone, which is not unusual in physics, the first step you should undertake is just to sit down and think for a while. This will save you a lot of time and effort later on. To emphasize the need for structuring in the software development process, the art of writing good programs is usually called software engineering. There are many specialized books in this fields, see e.g. Refs. [1, 2]. Here just the steps that should be undertaken to create a sophisticated software development process are stated. The following descriptions refer to the usual situation you find in physics: one or a few people are involved in the project. How to manage the development of big programs involving many developers is explained in literature.

\section{- Definition of the problem and solution strategies}

You should write down which problem you would like to solve. Drawing diagrams is always helpful! Discuss your problem with others and tell them how you would like to solve it. In this context many questions may appear, here some examples are given:

- What is the input you have to supply? In case you have only a few parameters, they can be passed to the program via options. In other cases, especially when chemical systems are to be simulated, many parameters have to be controlled and it may be advisable to use extra parameter files.

- Which results do you want to obtain and which quantities do you have to analyze? Very often it is useful to write the raw results of your simulations, e.g. the positions of all atoms or the orientations of all spins of your system, to a configuration file. The physical results can be obtained by post-processing. Then, in case new questions arise, it is very easy to analyze the data again. When using configuration files, you should estimate the amount of data you generate. Is there enough space on your disk? It may be helpful, to include the compression of the data files directly in your programs ${ }^{1}$.

- Can you identify "objects" in your problem? Objects may be physical entities like atoms or molecules, but also internal structures like nodes in a tree or elements of tables. Seeing the system and the program as a hierarchical collection of objects usually makes the problem easier to understand. More on object-oriented development can be found in Sec. 2.

\footnotetext{
${ }^{1}$ In $\mathrm{C}$ this can be achieved by calling system("gzip -f <filename $>$ "); after the file has been written and closed.
} 
- Is the program to be extended later on? Usually a code is "never" finished. You should foresee later extensions of the program and set up everything in a way it can be reused easily.

- Do you have existing programs available which can be included into the software project? If you have implemented your previous projects in the above mentioned fashion, it is very likely that you can recycle some code. But this requires experience and is not very easy to achieve at the beginning. But over the years you will have a growing library of programs which enables you to finish future software projects much quicker.

Has somebody else created a program which you can reuse? Sometimes you can rely on external code like libraries. Examples are the Numerical Recipes [3] and the LEDA library [4] which are covered in Sec. 5.

- Which algorithms are known? Are you sure that you can solve the problem at all? Many other techniques have been invented already. You should always search the literature for solutions which already exist. How searches can be simplified by using electronic data bases is covered more deeply in Sec. 9 .

Sometimes it is necessary to invent new methods. This part of a project may be the most time consuming.

\section{- Designing data structures}

Once you have identified the basic objects in your systems, you have to think about how to represent them in the code. Sometimes it is sufficient to define some struct types in $\mathrm{C}$ (or simple classes in $\mathrm{C}++$ ). But usually you will need to design a large set of data structures, referencing each other in a complicated way.

A sophisticated design of the data structures will lead to a better organized program, usually it will even run faster. For example, consider a set of vertices of a graph. Then assume that you have several lists $L_{i}$ each containing elements referencing the vertices of degree $i$. When the graph is altered in your program and thus the degrees of the vertices change, it is sometimes necessary to remove a vertex from one list and insert it into another. In this case you will gain speed, when your vertices data structures also contain pointers to the positions where they are stored in the lists. Hence, removing and inserting vertices in the lists will take only a constant amount of time. Without these additional pointers, the insert and delete operations have to scan partially through the lists to locate the elements, leading to a linear time complexity of these operations.

Again, you should perform the design of the data structures in a way, that later extensions are facilitated. For example when treating lattices of Ising spins, you should use data structures which are independent of the dimension or even of the structure of the lattice, an example is given in Sec. 4.1. 
When you are using external libraries, usually they have some data types included. The above mentioned LEDA library has many predefined data types like arrays, stacks, lists or graphs. You can have e.g. arrays of arbitrary objects, for example arrays of strings. Furthermore, it is possible to combine the data types in complicated ways, e.g. you can define a stack of graphs having strings attached to the vertices.

\section{- Defining small tasks}

After setting up the basic data types, you should think about which basic and complex operations, i.e. which subroutines, you need to manipulate the objects of your simulation. Since you have already thought a lot about your problem, you have a good overview, which operations may occur. You should break down the final task "perform simulation" into small subtasks, this means you use a top down approach in the design process. It is not possible to write a program in a sequential way as one code. For the actual implementation, a bottom up approach is recommended. This means you should start with the most basic operations. Later on you can use them to create more complicated operations. As always, you should define the subroutines in a way that they can be applied in a flexible way and extensions are easy to perform.

But it is not necessary that you must identify all basic operations at the beginning. During the development of the code, new applications may arise, which lead to the need for further operations. Also it may be required to change or extend the data structures defined before. However, the more you think in advance, the less you need to change the program later on.

As an example, the problem of finding ground states in Ising spin glasses via simulated annealing is considered. Some of basic operations are:

- Set up the data structures for storing the realizations of the interactions and for storing the spin glass configurations.

- Create a random realization of the interactions.

- Initialize a random spin configuration.

- Calculate the energy of a spin in the local field of its neighbors.

- Calculate the total energy of a system.

- Calculate the energy changes associated with a spin flip.

- Execute a Monte Carlo step.

- Execute a whole annealing run.

- Calculate the magnetization.

- Save a realization and corresponding spin configurations in a file.

It is not necessary to define a corresponding subroutine for all operations. Sometimes they require only a few numbers of lines in the code, like the 
calculation of the energy of one spin in the example above. In this case, such operations can be written directly in the code, or a macro (see Sec. 4.1) can be used.

\section{- Distributing work}

In case several people are involved in a project, the next step is to split up the work between the coworkers. If several types of objects appear in the program design, a natural approach is to make everyone responsible for one or several types of objects and the related operations. The code should be broken up into several modules (i.e. source files), such that every module is written by only one person. This makes the implementation easer and also helps testing the code (see below). Nevertheless, the partitioning of the work requires much care, since quite often some modules or data types depend on others. For this reason, the actual implementation of a data type should be hidden. This means that all interactions should be performed through exactly defined interfaces which do not depend on the internal representation, see also Sec. 2 on object-oriented programming.

When several people are editing the same files, which is usually necessary later on, even when initially each file was created by only one person, then you should use a source-code management system. It prevents several people from performing changes on the same file in parallel, which would cause a lot of trouble. Additionally, a source-code management system enables you to keep track of all changes made. An example of such a system is the Revision Control System (RCS), which is freely available through the GNU project [5] and part of the free operating system Linux.

\section{- Implementing the code}

With good preparation, the actual implementation becomes only a small part of the software development process. General style rules, guaranteeing clear structured code, which can even be understood several months later, are explained in Sec. 3. You should use a different file, i.e. a different module, for each coherent unit of data structures and subroutines; when using an object oriented language you should define different classes (see Sec. 2). This rule should be obeyed for the case of a one-person project as well. Large software projects containing many modules are easily maintained via makefiles (see Sec. 4.2).

Each subroutine and each module should be tested separately, before integrating many modules into one program. In the following some general hints concerning testing are presented.

\section{- Testing}

When performing tests on single subroutines, standard cases usually are used. This is the reason why many errors become apparent much later. Then, because the modules have already been integrated into one single program, errors are much harder to localize. For this reason, you should 
always try to find special and rare cases as well when testing a subroutine. Consider for example a procedure which inserts an element into a list. Then not only inserting in the middle of the list, but also at the beginning, at the end and into an empty list must be tested. Also, it is strongly recommended to read your code carefully once again before considering it finished. In this way many bugs can be found easily which otherwise must be tracked down by intensive debugging.

The actual debugging of the code can be performed by placing print instructions at selected positions in the code. But this approach is quite time consuming, because you have to modify and recompile your program several times. Therefore, it is advisable to use debugging tools like a source-code debugger and a program for checking the memory management. More about these tools can be found in Sec. 7. But usually you also need special operations which are not covered by an available tool. You should always write a procedure which prints out the current instance of the system that is simulated, e.g. the nodes and edges of a graph or the interaction constants of an Ising system. This facilitates the types of tests, which are described in the following.

After the raw operation of the subroutines has been verified, more complex tests can be performed. When e.g. testing an optimization routine, you should compare the outcome of the calculation for a small system with the result which can be obtained by hand. If the outcome is different from the expected result, the small size of the test system allows you to follow the execution of the program step by step. For each operation you should think about the expected outcome and compare it with the result originating from the running program.

Furthermore, it is very useful to compare the outcome of different methods applied to the same problem. For example, you know that there must be something wrong, in case an approximation method finds a better value than your "exact" algorithm. Sometimes analytical solutions are available, at least for special cases. Another approach is to use invariants. For example, when performing a Molecular Dynamics simulation of an atomic/molecular system (or a galaxy), energy and momentum must be conserved; only numerical rounding errors should appear. These quantities can be recorded very easily. If they change in time there must be a bug in your code. In this case, usually the formulas for the energy and the force are not compatible or the integration subroutine has a bug.

You should test each procedure, directly after writing it. Many developers have experienced that the larger the interval between implementation and tests is, the lower the motivation becomes for performing tests, resulting in more undetected bugs.

The final stage of the testing process occurs when several modules are integrated into one large running program. In the case where you are writing the code alone, not many surprises should appear, if you have performed 
many tests on the single modules. If several people are involved in the project, at this stage many errors occur. But in any case, you should always remember: there is probably no program, unless very small, which is bug free. You should know the following important result from theoretical computer science [6]: it is impossible to invent a general method, which can prove automatically that a given program obeys a given specification. Thus, all tests must be designed to match the current code.

In case a program is changed or extended several times, you should always keep the old versions, because it is quite common that by editing new bugs are introduced. In that case, you can compare your new code with the older version. Please note that editors like emacs only keep the second latest version as backup, so you have to take care of this problem yourself unless you use a source-code management system, where you are lucky, because it keeps all older version automatically.

For $\mathrm{C}$ programmers, it is always advisable to apply the -Wall (warning level: all) option. Then several bugs already show up during the compiling process, for example the common mistake to use '=' in comparisons instead of ' $==$ ', or the access to uninitialized variables ${ }^{2}$.

In $\mathrm{C}++$, some bugs can be detected by defining variables or parameter as const, when they are considered to stay unchanged in a block of code or subroutine. Here again, already the compiler will complain, if attempts to alter the value of such a variable are tried.

This part finishes with a warning: never try to save time when performing tests. Bugs which appear later on are much much harder to find and you will have to spend much more time than you have "saved" before.

\section{- Writing documentation}

This part of the software development process is very often disregarded, especially in the context of scientific research, where no direct customers exist. But even if you are using your own code, you should write good documentation. It should consist of at least three parts:

- Comments in the source code: You should place comments at the beginning of each module, in front of each subroutine or each selfdefined data structure, for blocks of the code and for selected lines. Additionally, meaningful names for the variables are crucial. Following these rules makes later changes and extension of the program much more straightforward. You will find in more hints on how a good programming style can be achieved Sec. 3 .

- On-line help: You should include a short description of the program, its parameters and its options in the main program. It should be printed, when the program is called with the wrong number/form of the parameters, or when the option -help is passed. Even when you

\footnotetext{
${ }^{2}$ But this is not true for some $\mathrm{C}++$ compilers when combining with option $-\mathrm{g}$.
} 
are the author of the program, after it has grown larger it is quite hard to remember all options and usages.

- External documentation: This part of the documentation process is important, when you would like to make the program available to other users or when it grows really complex. Writing good instructions is really a hard job. When you remember how often you have complained about the instructions for a video recorder or a word processor, you will understand why there is a high demand for good authors of documentation in industry.

\section{- Using the code}

Also the actual performance of the simulation usually requires careful preparation. Several question have to be considered, for example:

- How long will the different runs take? You should perform simulations of small systems and extrapolate to large system sizes.

- Usually you have to average over different runs or over several realizations of the disorder. The system sizes should also be chosen in a way that the number of samples is large enough to reduce the statistical fluctuations. It is better to have a reliable result for a small system than to treat only a few instances of a large system. If your model exhibits self averaging, the larger the sample, the less the number of samples can be. But, unfortunately, usually the numerical effort grows stronger than the system size, so there will be a maximum system size which can be treated with satisfying accuracy. To estimate the accuracy, you should always calculate the statistical error bar $\sigma(A)$ for each quantity $A^{3}$.

A good rule of a thumb is that each sample should take not more than 10 minutes. When you have many computers and much time available, you can attack larger problems as well.

- Where to put the results? In many cases you have to investigate your model for different parameters. You should organize the directories where you put the data and the names of the files in such a way that even years later the former results can be found quickly. You should put a README file in each directory, explaining what it contains.

If you want to start a sequence of several simulations, you can write a short script, which calls your program with different parameters within a loop.

- Logfiles are very helpful, where during each simulation some information about the ongoing processes are written automatically. Your program should put its version number and the parameters which have been used to start the simulation in the first line of each logfile. This allows a reconstruction of how the results have been obtained.

\footnotetext{
${ }^{3}$ The error bar is $\sigma(A)=\sqrt{\operatorname{Var}(A) /(N-1)}$, where $\operatorname{Var}(A)=\frac{1}{N} \sum_{i=1}^{N} a_{i}^{2}-\left(\frac{1}{N} \sum_{i=1}^{N} a_{i}\right)^{2}$ is the variance of the $N$ values $a_{1}, \ldots, a_{N}$.
} 
The steps given do not usually occur in linear order. It is quite common that after you have written a program and performed some simulations, you are not satisfied with the performance or new questions arise. Then you start to define new problems and the program will be extended. It may also be necessary to extend the data structures, when e.g. new attributes of the simulated models have to be included. It is also possible that a nasty bug is still hidden in the program, which is found later on during the actual simulations and becomes obvious by results which cannot be explained. In this case changes cannot be circumvented either.

In other words, the software development process is a cycle which is traversed several times. As a consequence, when planning your code, you should always keep this in mind and set up everything in a flexible way, so that extensions and code recycling can be performed easily.

\section{Object-oriented Software Development}

In recent years object-oriented programming languages like $\mathrm{C}++$, Smalltalk or Eiffel became very popular. But, using an object-oriented language and developing the program in an object-oriented style are not necessarily the same, although they are compatible. For example, you can set up your whole project by applying object-oriented methods even when using a traditional procedural programming language like C, Pascal or Fortran. On the other hand, it is possible to write very traditional programs with modern object-oriented languages. They help to organize your programs in terms of objects, but you have the flexibility to do it in another way as well. In general, taking an object-oriented viewpoint facilitates the analysis of problems and the development of programs for solving the problems. Introductions to object-oriented software development can be found e.g. in Refs. $[7,8,9]$. Here just the main principles are explained:

\section{- Objects and methods}

The real world is made of objects such as traffic-lights, books or computers. You can classify different objects according to some criteria into classes. This means different chairs belong to the class "chairs". The objects of many classes can have internal states, e.g. a traffic-light can be red, yellow or green. The state of a computer is much more difficult to describe. Furthermore, objects are useful for the environment, because other objects interact via operations with the object. You (belonging to the class "human") can read the state of a traffic light, some central computer may set the state or even switch the traffic light off.

Similar to the real world, you can have objects in programs as well. The internal state of an object is given by the values of the variables describing the object. Also it is possible to interact with the objects by calling subroutines (called methods in this context) associated with the objects.

Objects and the related methods are seen as coherent units. This means you define within one class definition the way the objects look, i.e. the data 
structures, together with the methods which access/alter the content of the objects. The syntax of the class definition depends on the programming language you use. Since implementational details are not relevant here, the reader is referred to the literature.

When you take the viewpoint of a pure object-oriented programmer, then all programs can be organized as collections of objects calling methods of each other. This is derived from the structure the real world has: it is a large set of interacting objects. But for writing good programs it is as in real life, taking an orthodox position imposes too many restrictions. You should take the best of both worlds, the object-oriented and the procedural world, depending on the actual problem.

\section{- Data capsuling}

When using a computer, you do not care about the implementation. When you press a key on the keyboard, you would like to see the result on the screen. You are not interested in how the key converts your pressing into an electrical signal, how this signal is sent to the input ports of the chips, how the algorithm treats the signal and so on.

Similarly, a main principle of object-oriented programming is to hide the actual implementation of the objects. Access to them is only allowed via given interfaces, i.e. via methods. The internal data structures are hidden, this is called private in $\mathrm{C}++$. The data capsuling has several advantages:

- You do not have to remember the implementation of your objects. When using them later on, they just appear as a black box fulfilling some duties.

- You can change the implementation later on without the need to change the rest of the program. Changes of the implementation may be useful e.g. when you want to increase the performance of the code or to include new features.

- Furthermore, you can have flexible data structures: several different types of implementations may coexist. Which one is chosen depends on the requirements. An example are graphs which can be implemented via arrays, lists, hash tables or in other ways. In the case of sparse graphs, the list implementation has a better performance. When the graph is almost complete, the array representation is favorable. Then you only have to provide the basic access methods, such as inserting/removing/testing vertices/edges and iterating over them, for the different internal representations. Therefore, higherlevel algorithms like computing a spanning tree can be written in a simple way to work with all internal implementations. When using such a class, the user just has to specify the representation he wants, the rest of the program is independent of this choice.

- Last but not least, software debugging is made easier. Since you have only defined ways the data can be changed, undesired side- 
effects become less common. Also the memory management can be controlled easier.

For the sake of flexibility, convenience or speed it is possible to declare internal variables as public. In this case they can be accessed directly from outside.

\section{- Inheritance}

- inheritance This means lower level objects can be specializations of higher level objects. For example the class of (German) "ICE trains" is a subclass of "trains" which itself is a subclass of "vehicles".

In computational physics, you may have a basic class of "atoms" containing mass, position and velocity, and built upon this a class of "charged atoms" by including the value of the charge. Then you can use the subroutines you have written for the uncharged atoms, like moving the particles or calculating correlation functions, for the charged atoms as well.

A similar form of hierarchical organization of objects works the other way round: higher level objects can be defined in terms of lower level objects. For example a book is composed of many objects belonging to the class "page". Each page can be regarded as a collection of many "letter" objects.

For the physical example above, when modeling chemical systems, you can have "atoms" as basic objects and use them to define "molecules". Another level up would be the "system" object, which is a collection of molecules.

\section{- Function/operator overloading}

This inheritance of methods to lower level classes is an example of operator overloading. It just means that you can have methods for different classes having the same name, sometimes the same code applies to several classes. This applies also to classes, which are not connected by inheritance. For example you can define how to add integers, real numbers, complex numbers or larger objects like lists, graphs or documents. In language like $\mathrm{C}$ or Pascal you can define subroutines to add numbers and subroutines to add graphs as well, but they must have different names. In $\mathrm{C}++$ you can define the operator "+" for all different classes. Hence, the operator-overloading mechanisms of object-oriented languages is just a tool to make the code more readable and clearer structured.

\section{- Software reuse}

Once you have an idea of how to build a chair, you can do it several times. Because you have a blueprint, the tools and the experience, building another chair is an easy task.

This is true for building programs as well: both data capsuling and inheritance facilitate the reuse of software. Once you have written your class for 
e.g. treating lists, you can include them in other programs as well. This is easy, because later on you do not have to care about the implementation. With a class designed in a flexible way, much time can be saved when realizing new software projects.

As mentioned before, for object-oriented programming you do not necessarily have to use an object-oriented language. It is true that they are helpful for the implementation and the resulting programs will look slightly more elegant and clear, but you can program everything with a language like $\mathrm{C}$ as well. In $\mathrm{C}$ an object-oriented style can be achieved very easily. As an example a class histo implementing histograms is outlined, which are needed for almost all types of computer simulations as evaluation and analysis tools.

First you have to think about the data you would like to store. That is the histogram itself, i.e. an array table of bins. Each bin just counts the number of events which fall into a small interval. To achieve a high degree of flexibility, the range and the number of bins must be variable. From this, the width delta of each bin can be calculated. For convenience delta is stored as well. To count the number of events which are outside the range of the table, the entries low and high are introduced. Furthermore, statistical quantities like mean and variance should be available quickly and with high accuracy. Thus, several summarized moments sum of the distribution are stored separately as well. Here the number of moments_HISTO_NOM_ is defined as a macro, converting this macro to variable is straightforward. All together, this leads to the following $\mathrm{C}$ data structure:

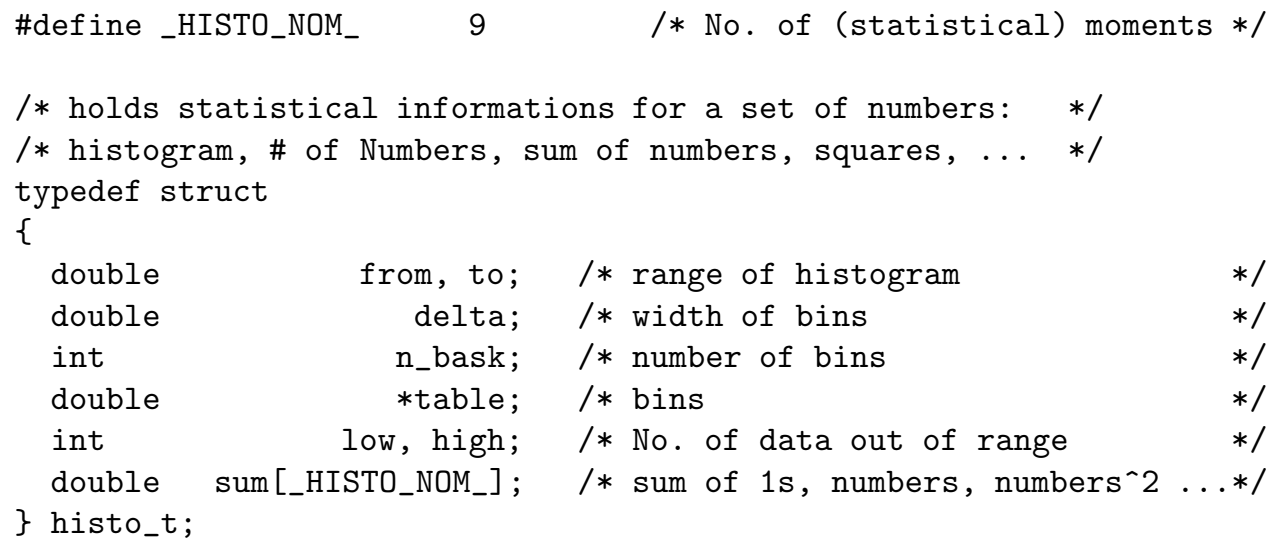

Here, the postfix _t is used to stress the fact that the name histo_t denotes a type. The bins are double variables, which allows for more general applications. Please note that it is still possible to access the internal structures from outside, but it is not necessary and not recommended. In $\mathrm{C}++$, you could prevent this by declaring the internal variables as private. Nevertheless, everything can be done via special subroutines. First of all one must be able to create and delete histograms, please note that some simple error-checking is included in the program: 


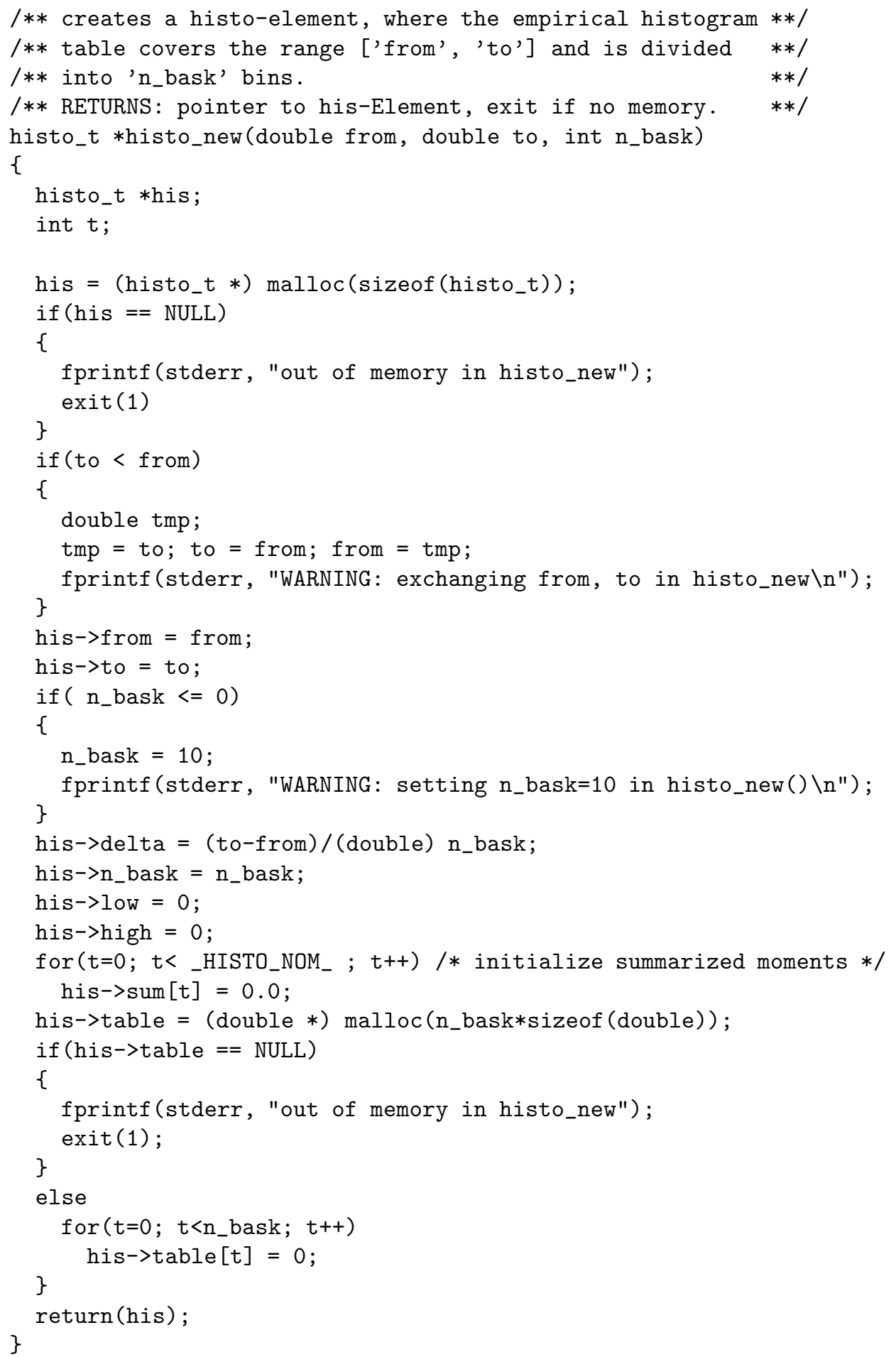




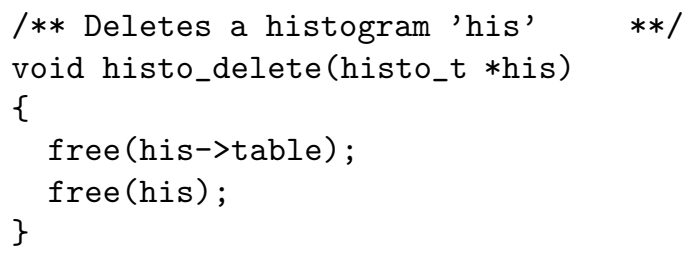

All histogram objects are created dynamically by calling histo_new(), this corresponds to a call of the constructor or new in $\mathrm{C}++$. The objects are addressed via pointers. Whenever a method, i.e. a procedure in $\mathrm{C}$, of the histo class is called, the first argument will always be a pointer to the corresponding histogram. This looks slightly less elegant than writing histo.method() in $\mathrm{C}++$, but it is really the same. When avoiding direct access, the realization using $\mathrm{C}$ is perfectly equivalent to $\mathrm{C}++$ or other object-oriented languages. Inheritance can be implemented, by including pointers to histo_t objects in other type definitions. When these higher level objects are created, a call to histo_new() must be included, while a call to histo_delete(), corresponding to the destructor in $\mathrm{C}++$, is necessary, to implement a correct deletion of the more complex objects. As a final example, the procedures for inserting an element into the table and calculating the mean are presented. It is easy to figure out how other subroutines for e.g. calculating the variance/higher moments or printing a histogram can be realized. The complete library can be obtained for free [10].

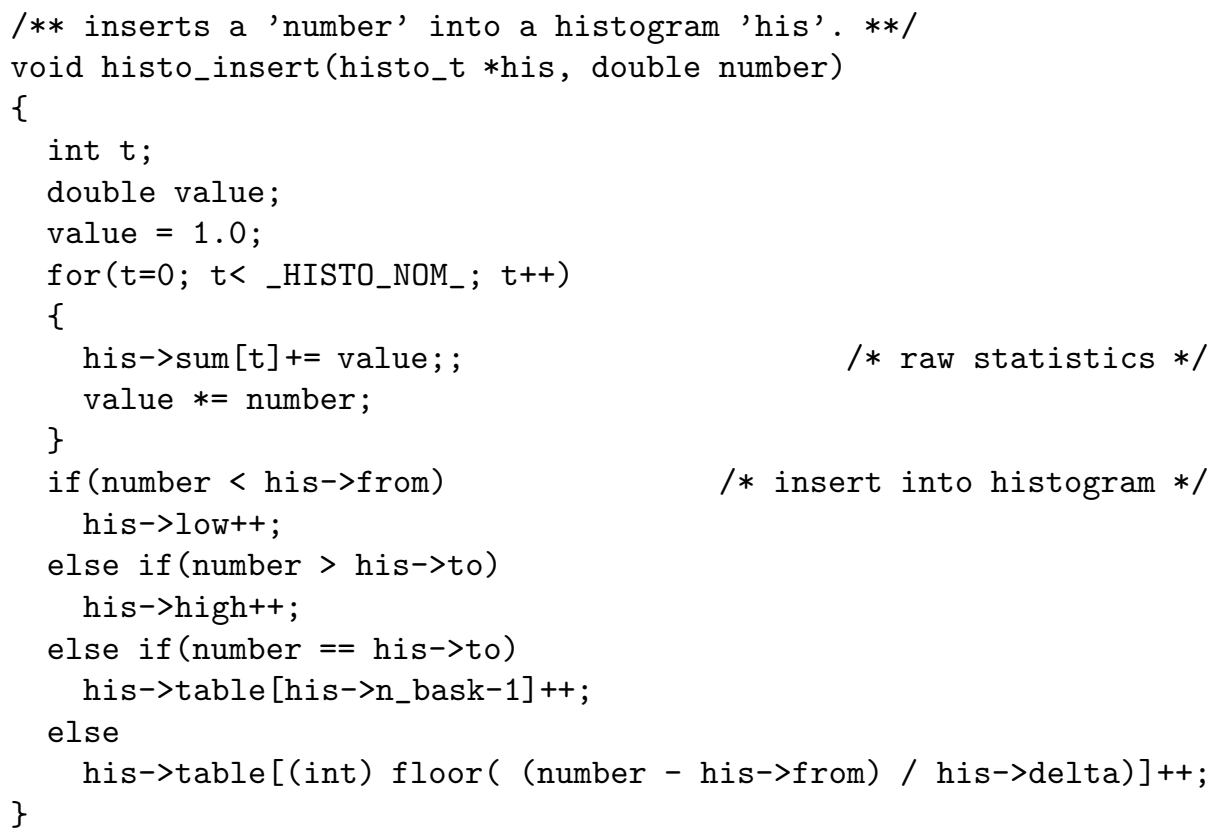




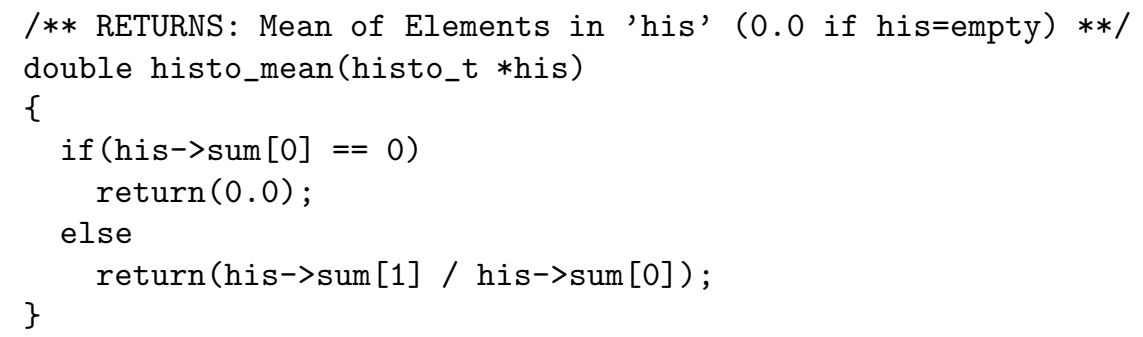

\section{Programming Style}

The code should be written in a style that enables the author, and other people as well, to understand and modify the program even years later. Here briefly some principles you should follow are stated. Just a general style of description is given. Everybody is free to choose his/her own style, as long as it is precise and consistent.

- Split your code into several modules. This has several advantages:

- When you perform changes, you have to recompile only the modules which have been edited. Otherwise, if everything is contained in a long file, the whole program has to be recompiled each time again.

- Subroutines which are related to each other can be collected in single modules. It is much easier to navigate in several short files than in one large program.

- After one module has been finished and tested it can be used for other projects. Thus, software reuse is facilitated.

- Distributing the work among several people is impossible if everything is written into one file. Furthermore, you should use a sourcecode management system (see Sec. 1) in case several people are involved in avoiding uncontrolled editing.

- To keep your program logically structured, you should always put data structures and implementations of the operations in separate files. In $\mathrm{C} / \mathrm{C}++$ this means you have to write the data structures in a header $(. \mathrm{h})$ file and the code into a source code (.c/ .cpp) file.

- Try to find meaningful names for your variables and subroutines. Therefore, during the programming process it is much easier to remember their meanings, which helps a lot in avoiding bugs. Additionally, it is not necessary to look up the meaning frequently. For local variables like loop counters, it is sufficient and more convenient to have short (e.g. one letter) names.

In the beginning this might seem to take additional time (writing e.g. 'kinetic_energy' for a variable instead of 'x10'). But several months 
after you have written the program, you will appreciate your effort, when you read the line

kinetic_energy $+=0.5 *$ atom [i].mass*atom[i].veloc*atom [i].veloc;

instead of

$\mathrm{x} 10+=0.5 * x 34[i] . \mathrm{a} * \mathrm{x} 34[\mathrm{i}] . \mathrm{b} * \mathrm{x} 34[\mathrm{i}] . \mathrm{b} ;$

- You should use proper indentation of your lines. This helps a great deal in recognizing the structure of a program. Many bugs are caused by misaligned braces forming a block of code. Furthermore, you should place at most one command per line of code. The reader will probably agree that

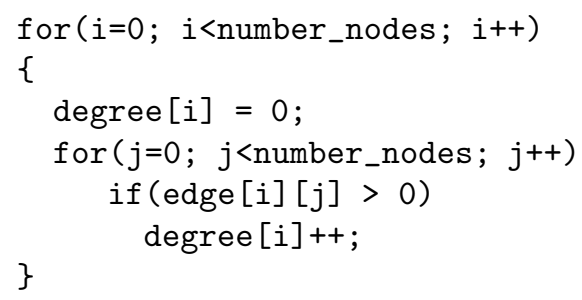

is much faster to understand than

for (i=0; i<number_nodes; $i++)\{$ degree $[i]=0$; for $(j=0$;

$j<$ number_nodes; $j++)$ if (edge $[i][j]>0)$ degree $[i]++;\}$

- Avoid jumping to other parts of a program via the "goto" command. This is bad style originating from programming in assembler or BASIC. In modern programming languages, for every logical programming construct there are corresponding commands. "Goto" commands make a program harder to understand and much harder to debug if it does not work as it should.

In case you want to break out of a loop, you can use a while/until loop with a flag that indicates if the loop is to be stopped. In C, if you are lazy, you can use the commands break or continue.

- Do not use global variables. At first sight the use of global variables may seem tempting: you do not have to care about parameters for subroutines, everywhere the variables are accessible and everywhere they have the same name. Programming is done much faster.

But later on you will have a bad time: many bugs are created by improper use of global variables. When you want to check for a definition of a variable you have to search the whole list of global variables, instead of 
just checking the parameter list. Sometimes the range of validity of a global variable is overwritten by a local variable. Furthermore, software re-usage is almost impossible with global variables, because you always have to check all variables used in a module for conflicts and you are not allowed to employ the name for another object. When you want to pass an object to a subroutine via a global variable, you do not have the choice of how to name the object which is to be passed. Most important, when you have a look onto a subroutine after some months, you cannot see immediately which objects are changed in the subroutine, instead you will have to read the whole subroutine again. If you avoid this practice, you just have to look at the parameter list. Finally, when a renaming occurs, you have to change the name of a global variable everywhere in the whole program. Local variables can be changed with little effort.

- Finally, an issue of utmost importance: Do not be economical with comments in your source code! Most programs, which may appear logically structured when writing them, will be a source of great confusion when being read some weeks later. Every minute you spend on writing reasonable comments you will save later on several times over. You should consider different types of comments.

- Module comments: At the beginning of each module you should state its name, what the module does, who wrote it and when it was written. It is a useful practice to include a version history, which lists the changes that have been performed. A module comment might look like this:

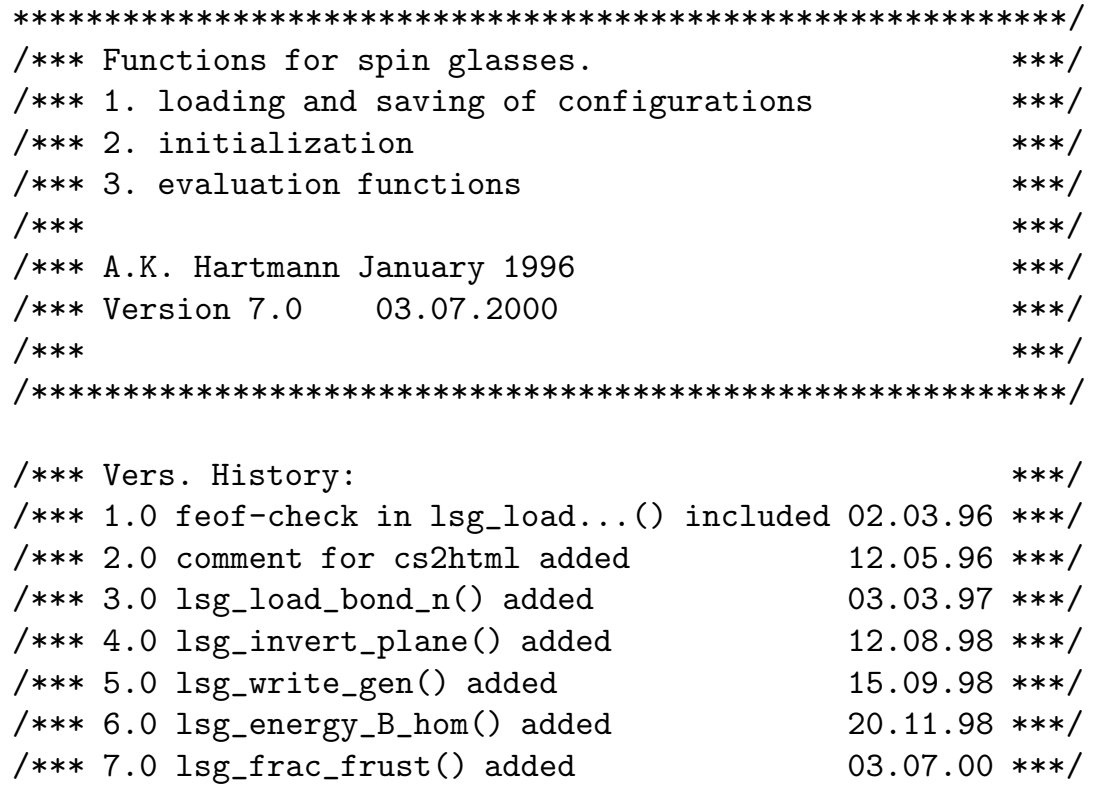


- Type comments: For each data type (a struct in $\mathrm{C}$ or class in $\mathrm{C}++$ ) which you define in a header file, you should attach several lines of comments describing the data type's structure and its application. For a class definition, also the methods which are available should be described. Furthermore, for a structure, each element should be explained. A nice arrangement of the comments makes everything more readable. An example of what such a comment may look like can be seen in Sec. 2 for the data type histo_t.

- Subroutine comments: For each subroutine, its purpose, the meaning of the input and output variables and the preconditions which have to be fulfilled before calling must be stated. In case you are lazy and do not write a man page, a comment atop of a subroutine is the only source of information, should you want to use the subroutine later on in another program.

If you use some special mathematical methods or clever algorithms in the subroutine, you should always cite the source in the comment. This facilitates later on the understanding of how the methods works.

The next example shows what the comment for a subroutine may look like:

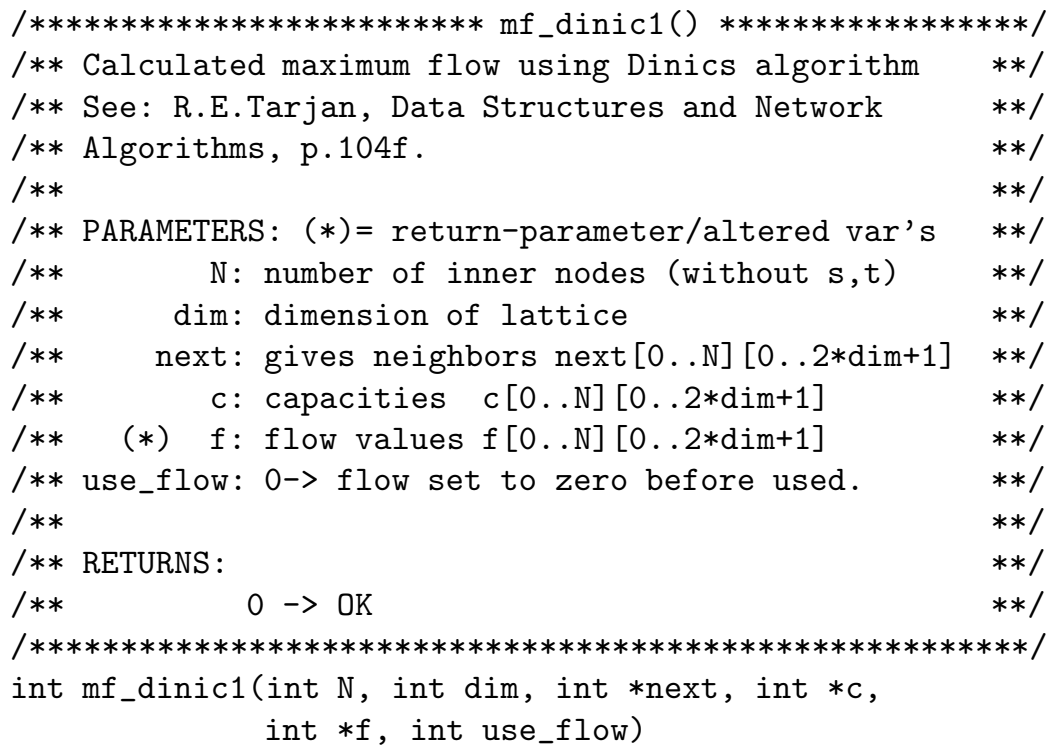

- Block comments: You should divide each subroutine, unless it is very short, into several logical blocks. A rule of thumb is that no block should be longer than the number of lines you can display in your editor window. Within one or two lines you should explain what is done in the block. Example:

/* go through all nodes except source s and sink t in */
/* reversed topological order and set capacities 
for (t2=num_nodes $-2 ;$ t2 $>0 ;$ t2-- )

...

- Line comments: They are the lowest level comments. Since you are using (hopefully) sound names for data types, variables and subroutines, many lines should be self explanatory. But in case the meaning is not obvious, you should add a small comment at the end of a line, for example:

$$
\mathrm{C}(\mathrm{t}, \mathrm{SOURCE})=\operatorname{cap} \_s 2 \mathrm{t}[\mathrm{t}] ; \quad / * \text { restore capacities } * /
$$

Aligning all comments to the right makes a code easier to read. Please avoid unnecessary comments like

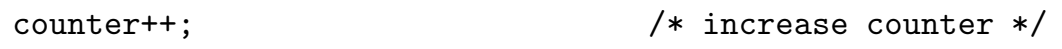

or unintelligible comments like

$$
\text { minimize_energy (spin, } N \text {, next, 5); /* I try this one } * /
$$

The line containing $\mathrm{C}(\mathrm{t}$, SOURCE) is an example of the application of a macro. This subject is covered in the following section.

\section{Programming Tools}

Programming languages and UNIX/Linux offer many concepts and tools which help you to perform large simulation projects. Here, three of them are presented: macros, which are explained first, makefiles and scripts.

\subsection{Using Macros}

Macros are shortcuts for code sequences in programming languages. Their primary purpose is to allow computer programs to be written more quickly. But the main benefit comes from the fact that a more flexible software development becomes possible. By using macros appropriately, programs become better structured, more generally applicable and less error-prone. Here it is explained how macros are defined and used in $\mathrm{C}$, a detailed introduction can be found in $\mathrm{C}$ textbooks such as Ref. [11]. Other high-level programming languages exhibit similar features.

In $\mathrm{C}$ a macro is constructed via the \#define directive. Macros are processed in the preprocessing stage of the compiler. This directive has the form

\#define name definition

Each definition must be on one line, without other definitions or directives. If the definition extends over more than one line, each line except the last one has to be ended with the backslash \symbol. The simplest form of a macro is a constant, e.g.

\#define PI 3.1415926536 
You can use the same sorts of names for macros as for variables. It is convention to use only upper-case letters for macros. A macro can be deleted via the \#undef directive.

When scanning the code, the preprocessor just replaces literally every occurrence of a macro by its definition. If you have for example the expression $2.0 * \mathrm{PI} *$ omega in your code, the preprocessor will convert it into $2.0 * 3.1415926536 *$ omega. You can use macros also in the definition of other macros. But macros are not replaced in strings, i.e. printf ("PI") ; will print PI and not 3.1415926536 when the program is running.

It is possible to test for the (non)existence of macros using the \#ifdef and \#ifndef directives. This allows for conditional compiling or for platformindependent code, such as e.g. in

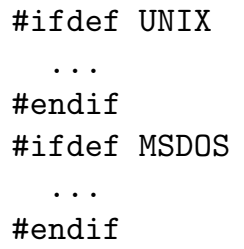

Please note that it is possible to supply definitions of macros to the compiler via the $-D$ option, e.g. gcc -o program program.c -DUNIX=1. If a macro is used only for conditional \#ifdef/\#ifndef statements, an assignment like $=1 \mathrm{can}$ be omitted, i.e. -DUNIX is sufficient.

When programs are divided into several modules, or when library functions are used, the definition of data types and functions are provided in header files (.h files). Each header file should be read by the compiler only once. When projects become more complex, many header files have to be managed, and it may become difficult to avoid multiple scanning of some header files. This can be prevented automatically by this simple construction using macros:

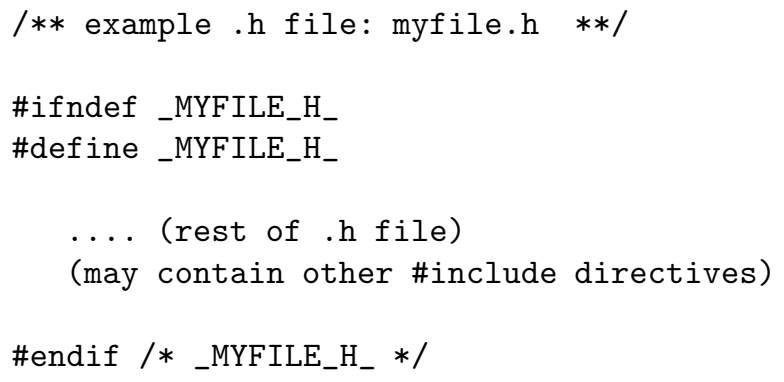

After the body of the header file has been read the first time during a compilation process, the macro _MYFILE_H_ is defined, thus the body will never read be again.

So far, macros are just constants. You will benefit from their full power when using macros with arguments. They are given in braces after the name of the macro, such as e.g. in 
\#define $\operatorname{MIN}(\mathrm{x}, \mathrm{y}) \quad((\mathrm{x})<(\mathrm{y}) ?(\mathrm{x}):(\mathrm{y}))$

You do not have to worry more than usual about the names you choose for the arguments, there cannot be a conflict with other variables of the same name, because they are replaced by the expression you provide when a macro is used, e.g. $\operatorname{MIN}(4 * a, b-32)$ will be expanded to $(4 * a)<(b-32)$ ? $(4 * a):(b-32)$.

The arguments are used in braces () in the macro, because the comparison $<$ must have the lowest priority, regardless which operators are included in the expressions that are supplied as actual arguments. Furthermore, you should take care of unexpected side effects. Macros do not behave like functions. For example when calling MIN $(\mathrm{a}++, \mathrm{b}++)$ the variable $\mathrm{a}$ or $\mathrm{b}$ may be increased twice when the program is executed. Usually it is better to use inline functions (or sometimes templates in $\mathrm{C}++$ ) in such cases. But there are many applications of macros, which cannot be replaced by incline functions, like in the following example, which closes this section.

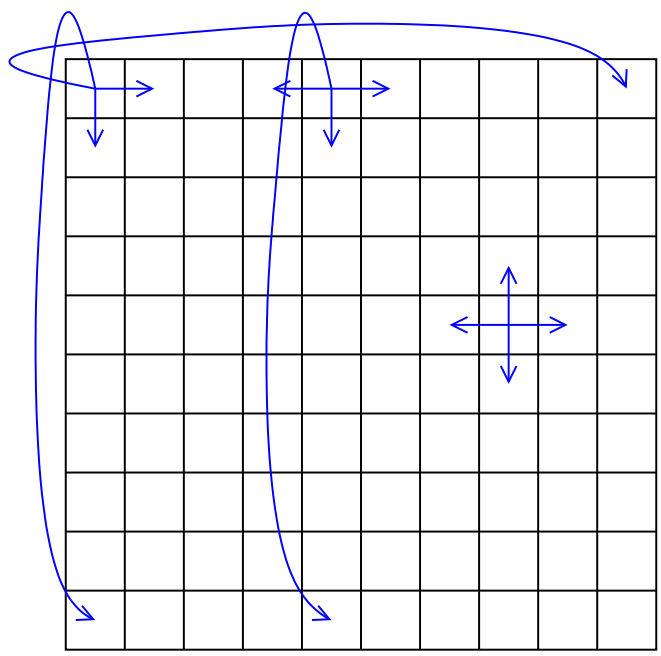

Figure 1: A square lattice of size $10 \times 10$ with periodical boundary conditions. The arrows indicate the neighbors of the spins.

The example illustrates how a program can be written in a clear way using macros, making the program less error-prone, and furthermore allowing for a broad applicability. A system of Ising spins is considered, that is a lattice where at each site $i$ a particle $\sigma_{i}$ is placed. Each particle can have only two states $\sigma_{i}= \pm 1$. It is assumed that all lattice sites are numbered from 1 to $N$. This is different from $\mathrm{C}$ arrays, which start at index 0 , the benefit of starting with index 1 for the sites will become clear below. For the simplest version of the model only neighbors of spins are interacting. With a two-dimensional square lattice of size $N=L \times L$ a spin $i$, which is not at the boundary, interacts with spins $i+1$ ( $+x$-direction), $i-1(-x$-direction $), i+L(+y$-direction $)$ and $i-L(-y$ - 
direction). A spin at the boundary may interact with fewer neighbors when free boundary conditions are assumed. With periodic boundary conditions (pbc), all spins have exactly 4 neighbors. In this case, a spin at the boundary interacts also with the nearest mirror images, i.e. with the sites that are neighbors if you consider the system repeated in each direction. For a $10 \times 10$ system spin 5 , which is in the first row, interacts with spins $5+1=6,5-1=4,5+10=15$ and through the pbc with spin 95, see Fig. 1. The spin in the upper left corner, spin 1 , interacts with spins $2,11,10$ and 91 . In a program pbc can be realized by performing all calculations modulo $L$ (for the $\pm x$-directions) and modulo $L^{2}$ (for the $\pm y$-directions), respectively.

This way of realizing the neighbor relations in a program has several disadvantages:

- You have to write the code everywhere where the neighbor relation is needed. This makes the source code larger and less clear.

- When switching to free boundary conditions, you have to include further code to check whether a spin is at the boundary.

- Your code works only for one lattice type. If you want to extend the program to lattices of higher dimension you have to rewrite the code or provide extra tests/calculations.

- Even more complicated would be an extension to different lattice structures such as triangle or face-center cubic. This would make the program look even more confusing.

An alternative is to write the program directly in a way it can cope with almost arbitrary lattice types. This can be achieved by setting up the neighbor relation in one special initialization subroutine (not discussed here) and storing it in an array next []. Then, the code outside the subroutine remains the same for all lattice types and dimensions. Since the code should work for all possible lattice dimensions, the array next is one dimensional. It is assumed that each site has

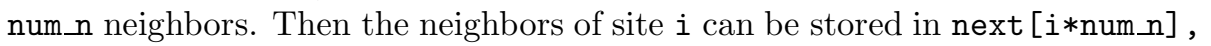
next $\left[i * n u m \_n+1\right], \ldots$, next $\left[i * n u m \_n+n u m \_n-1\right]$. Please note that the sites are numbered beginning with 1 . This means, a system with $\mathrm{N}$ spins needs an array NEXT of size $(\mathrm{N}+1) *$ num $n$. When using free boundary conditions, missing neighbors can be set to 0 . The access to the array can be made easier using a macro NEXT:

\#define NEXT $(i, r) \quad$ next $\left[(i) * n u m \_n+r\right]$

$\operatorname{NEXT}(i, r)$ contains the neighbor of spin $i$ in direction $r$. For e.g. a quadratic system, $\mathrm{r}=0$ is the $+x$-direction, $\mathrm{r}=1$ the $-x$-direction, $\mathrm{r}=2$ the $+y$-direction and $\mathrm{r}=3$ the $-y$-direction. However, which convention you use depends on you, but you should make sure you are consistent. For the case of a quadratic lattice, it is num_n=4. Please note that whenever the macro NEXT is used, there must be a variable num_n defined, which stores the number of neighbors. You could 
include num_n as a third parameter of the macro, but in this case a call of the macro looks slightly more confusing. Nevertheless, the way you define such a macro depends on your personal preferences.

Please note that the NEXT macro cannot be realized by an inline function, in case you want to set values directly like in $\operatorname{NEXT}(i, 0)=i+1$. Also, when using an inline function, you would have to include all parameters explicitly, i.e. num_n in the example. The last requirement could be circumvented by using global variables, but this is bad programming style as well.

When the system is an Ising spin glass, the sign and magnitude of the interaction may be different for each pair of spins. The interaction strengths can be stored in a similar way to the neighbor relation, e.g. in an array $j[]$. The access can be simplified via the macro $J$ :

\#define $J(i, r) \quad j\left[(i) * n u m \_n+r\right]$

A subroutine for calculating the energy $H=\sum_{\langle i, j\rangle} J_{i j} \sigma_{i} \sigma_{j}$ may look as follows, please note that the parameter $\mathrm{N}$ denotes the number of spins and the values of the spins are stored in the array sigma []:

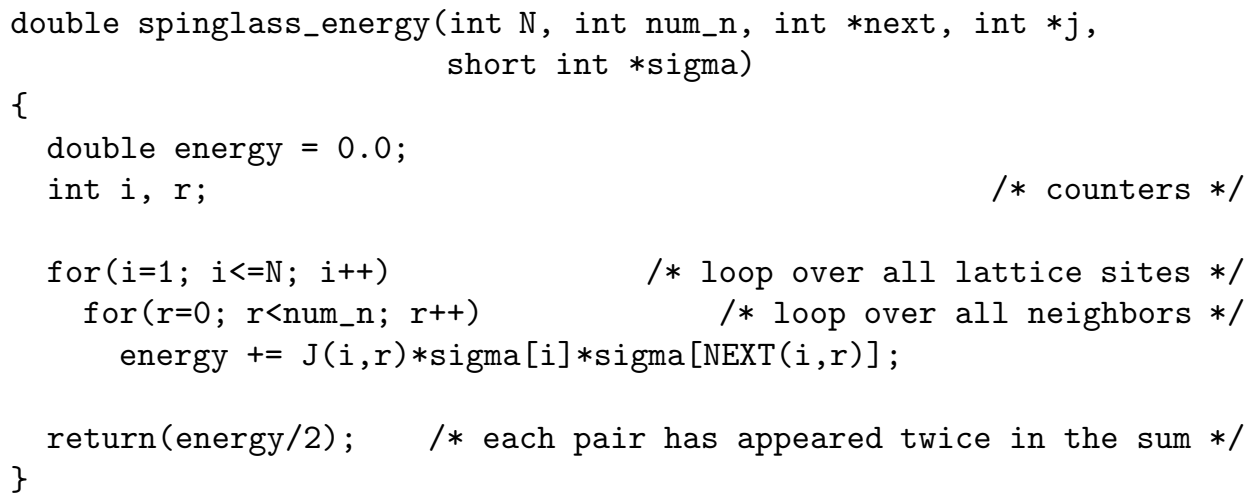

For this piece of code the comments explaining the parameters and the purpose of the code are just missing for convenience. In the actual program it should be included.

The code for spinglass_energy ( ) is very short and clear. It works for all kinds of lattices. Only the subroutine where the array next [] is set up has to be rewritten when implementing a different type of lattice. This is true for all kinds of code realizing e.g. a Monte Carlo scheme or the calculation of a physical quantity. For free boundary conditions, additionally sigma [0] =0 must be assigned to be consistent with the convention that missing neighbors have the id 0 . This is the reason, why the spin site numbering starts with index 1 while $\mathrm{C}$ arrays start with index 0 .

\subsection{Make Files}

If your software project grows larger, it will consist of several source-code files. Usually, there are many dependencies between the different files, e.g. a data 
type defined in one header file can be used in several modules. Consequently, when changing one of your source files, it may be necessary to recompile several parts of the program. In case you do not want to recompile your files every time by hand, you can transfer this task to the make tool which can be found on UNIX operating systems. A complete description of the abilities of make can be found in Ref. [12]. You should look on the man page (type man make) or in the texinfo file [13] as well. For other operating systems or software development environments, similar tools exists. Please consult the manuals in case you are not working with a UNIX type of operating system.

The basic idea of make is that you keep a file which contains all dependencies between your source code files. Furthermore, it contains commands (e.g. the compiler command) which generate the resulting files called targets, i.e. the final program and/or object (.o) files. Each pair of dependencies and commands is called rule. The file containing all rules of a project is called makefile, usually it is named Makefile and should be placed in the directory where the source files are stored.

A rule can be coded by two lines of the form

$$
\begin{aligned}
& \text { target : sources } \\
& <\text { tab }>\text { command }(s)
\end{aligned}
$$

The first line contains the dependencies, the second one the commands. The command line must begin with a tabulator symbol $\langle t a b\rangle$. It is allowed to have several targets depending on the same sources. You can extend the lines with the backslash "V" at the end of each line. The command line is allowed to be left empty. An example of a dependency/command pair is

simulation.o: simulation.c simulation.h

<tab> cc -c simulation.c

This means that the file simulation. o has to be compiled if either simulation.c or simulation.h have been changed. The make program is called by typing make on the command line of a UNIX shell. It uses the date of the last changes, which is stored along with each file, to determine whether a rebuild of some targets is necessary. Each time at least one of the source files are newer than the corresponding target files, the commands given after the $\langle$ tab $\rangle$ are called. Specifically, the command is called, if the target file does not exist at all. In this special case, no source files have to be given after the colon in the first line of the rule.

It is also possible to generate meta rules, which e.g. tell how to treat all files which have a specific suffix. Standard rules, how to treat files ending for example with .c are already included, but can be changed for each file by stating a different rule. This subject is covered in the man page of make.

The make tool always tries to build only the first object of your makefile, unless enforced by the dependencies. Hence, if you have to build several independent object files object1, object2, object 3 , the whole compiling must be toggled by the first rule, thus your makefile should read like this 


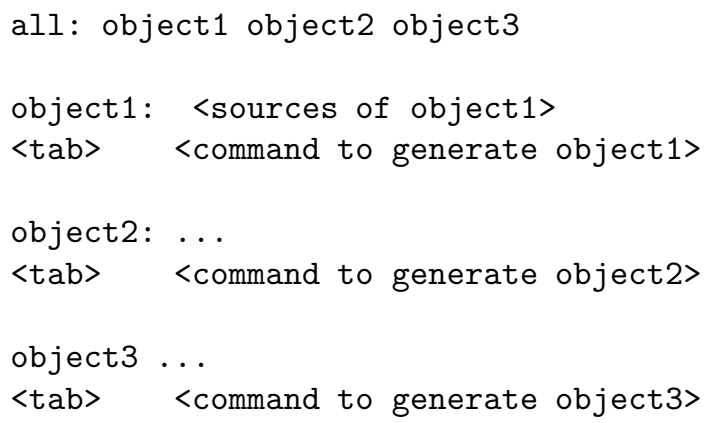

It is not necessary to separate different rules by blank lines. Here it is just for better readability. If you want to rebuild just e.g. object3, you can call make object3. This allows several independent targets to be combined into one makefile. When compiling programs via make, it is common to include the target "clean" in the makefile such that all objects files are removed when make clean is called. Thus, the next call of make (without further arguments) compiles the whole program again from scratch. The rule for 'clean' reads like

clean:

$\langle t a b\rangle \quad r m-f * . o$

Also iterated dependencies are allowed, for example

object1: object2

object2: object3

$\langle t a b\rangle \ldots$

object3: ...

$\langle t a b\rangle \ldots$

The order of the rules is not important, except that make always starts with the first target. Please note that the make tool is not just intended to manage the software development process and toggle compile commands. Any project where some output files depend on some input files in an arbitrary way can be controlled. For example you could control the setting of a book, where you have text-files, figures, a bibliography and an index as input files. The different chapters and finally the whole book are the target files.

Furthermore, it is possible to define variables, sometimes also called macros. They have the format

$$
\text { variable=definition }
$$

Also variables belonging to your environment like \$HOME can be referenced in the makefile. The value of a variable can be used, similar to shells variables, by placing a $\$$ sign in front of the name of the variable, but you have to embrace 
the name by $(\ldots)$ or $\{\ldots\}$. There are some special variables, e.g. $\$ @$ holds the name of the target in each corresponding command line, here no braces are necessary. The variable CC is predefined to hold the compiling command, you can change it by including for example

$\mathrm{CC}=\mathrm{gcc}$

in the makefile. In the command part of a rule the compiler is called via $\$(\mathrm{CC})$. Thus, you can change your compiler for the whole project very quickly by altering just one line of the makefile.

Finally, it will be shown what a typical makefile for a small software project might look like. The resulting program is called simulation. There are two additional modules init.c, run.c and the corresponding header .h files. In datatypes.h types are defined which are used in all modules. Additionally, an external precompiled object file analysis . o in the directory $\$ H O M E / 1 i b$ is to be linked, the corresponding header file is assumed to be stored in \$HOME/include. For init.o and run.o no commands are given. In this case make applies the predefined standard command for files having . o as suffix, which reads like

$<t a b>\quad \$(C C) \quad \$(C F L A G S)-c \quad \$ @$

where the variable CFLAGS may contain options passed to the compiler and is initially empty. The makefile looks like this, please note that lines beginning with "\#" are comments.

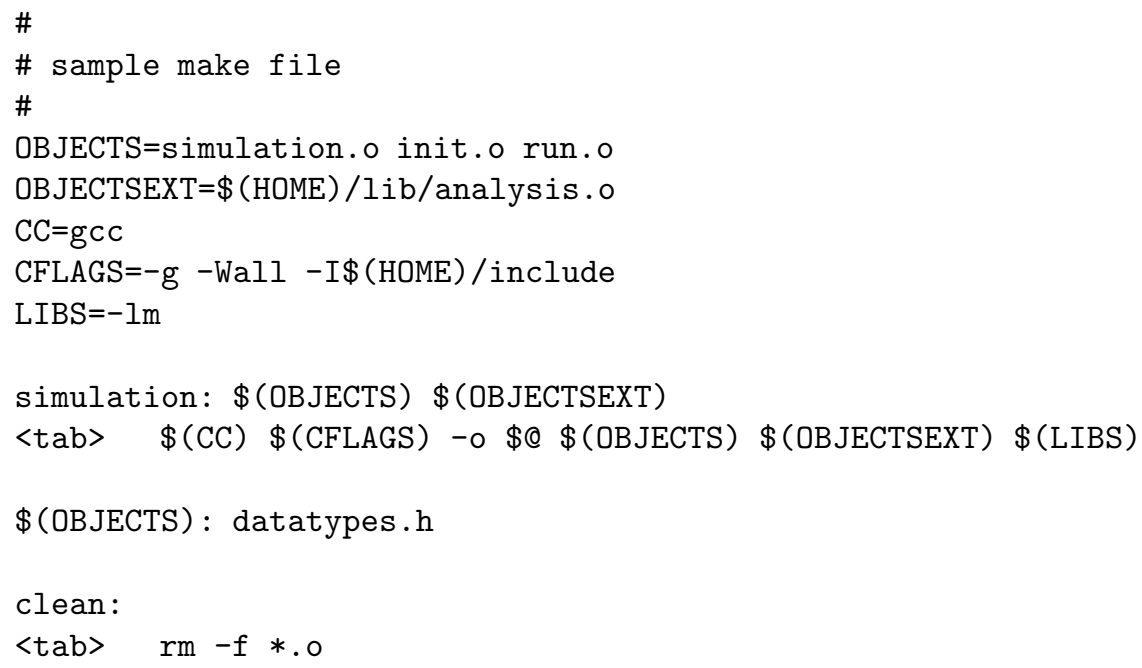

The first three lines are comments, then five variables OBJECTS, OBJECTSEXT, CC, CFLAGS and LIBS are assigned. The final part of the makefile are the rules. Please note that sometimes bugs are introduced, if the makefile is incomplete. For example consider a header file which is included in several code files, but this is not mentioned in the makefile. Then, if you change e.g. a data type in the 
header file, some of the code files might not be compiled again, especially those you did not change. Thus the same objects files can be treated with different formats in your program, yielding bugs which seem hard to explain. Hence, in case you encounter mysterious bugs, a make clean might help. But most of the time, bugs which are hard to explain are due to errors in your memory management. How to track down those bugs is explained in Sec. 7.

The make tool exhibits many other features. For additional details, please consult the references given above.

\subsection{Scripts}

Scripts are even more general tools than make files. They are in fact small programs, but they are usually not compiled, i.e. they are quickly written but they run slowly. Scripts can be used to perform many administration tasks like backing up data, installing software or running simulation programs for many different parameters. Here only an example concerning the last task is presented. For a general introduction to scripts, please refer to a book on UNIX/Linux.

Assume that you have a simulation program called coversim21 which calculates vertex covers of graphs. In case you do not know what a vertex cover is, it does not matter, just regard it as one optimization problem characterized by some parameters. You want to run the program for a fixed graph size L, for a fixed concentration $\mathrm{c}$ of the edges, average over num realizations and write the results to a file, which contains a string appendix in its name to distinguish it from other output files. Furthermore, you want to iterate over different relative sizes $\mathrm{x}$. Then you can use the following script run.scr:

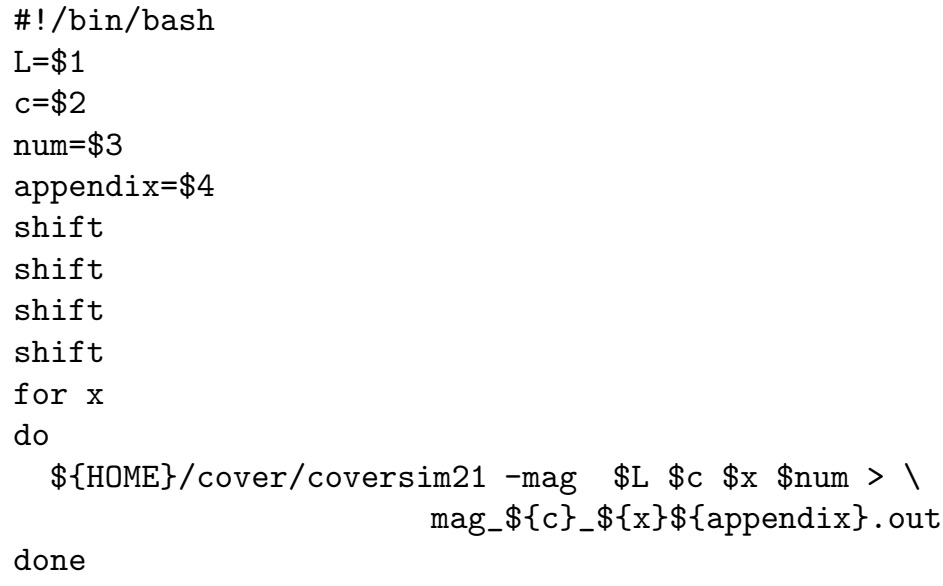

The first line starting with "\#" is a comment line, but it has a special meaning. It tells the operating system the language in which the script is written. In this case it is for the bash shell, the absolute pathname of the shell is given. Each UNIX shell has its own script language, you can use all commands which are allowed in the shell. There are also more elaborate script languages like perl or phyton, but they are not covered here. 
Scripts can have command line arguments, which are referred via $\$ 1, \$ 2, \$ 2$ etc., the name of the script itself is stored in $\$ 0$. Thus, in the lines 2 to 5 , four variables are assigned. In general, you can use the arguments everywhere in the script directly, i.e. it is not necessary to store them in other variables. It is done here because in the next four lines the arguments $\$ 1$ to $\$ 4$ are thrown away by four shift commands. Then, the argument which was on position five at the beginning is stored in the first argument. Argument zero, containing the script name, is not affected by the shift.

Next, the script enters a loop, given by "for $\mathrm{x}$; do ... done". This construction means that iteratively all remaining arguments are assigned to the variable " $\mathrm{x}$ " and each time the body of the loop is executed. In this case, the simulation is started with some parameters and the output directed to a file. Please note that you can state the loop parameters explicitly like in "for size in 10204080 160; do ... done".

The above script can be called for example by

run.scr $100 \quad 0.51000$ testA $0.20 \quad 0.22 \quad 0.24 \quad 0.26 \quad 0.28 \quad 0.30$

which means that the graph size is 100 , the fraction of edges is 0.5 , the number of realizations per run is 100 , the string testA appears in the output file name and the simulation is performed for the relative sizes $0.20,0.22,0.24,0.26,0.28$, 0.30 .

\section{$5 \quad$ Libraries}

Libraries are collections of subroutines and data types, which can be used in other programs. There are libraries for numerical methods such as integration or solving differential equations, for storing, sorting and accessing data, for fancy data types like lists or trees, for generating colorful graphics and for thousands of other applications. Some can be obtained for free, while other, usually specialized libraries have to be purchased. The use of libraries speeds up the software development process enormously, because you do not have to implement every standard method by yourself. Hence, you should always check whether someone has done the jobs for you already, before starting to write a program. Here, two standard libraries are briefly presented, providing routines which are needed for most computer simulations.

Nevertheless, sometimes it is inevitable to implement some methods by yourself. In this case, after the code has been proven to be reliable and useful for some time, you can put it in a self-created library. How to create libraries is explained in the last part of this section.

\subsection{Numerical Recipes}

The Numerical Recipes (NR) [3] contain a huge number of subroutines to solve standard numerical problems. Among them are: 
- solving linear equations

- performing interpolations

- evaluation and integration of functions

- solving nonlinear equations

- minimizing functions

- diagonalization of matrices

- Fourier transform

- solving ordinary and partial differential equations.

The algorithms included are all state of the art. There are several libraries dedicated to similar problems, e.g. the library of the Numerical Algorithms Group [14] or the subroutines which are included with the Maple software package [15]. To give you an impression how the subroutines can be used, just a short example is presented. Consider the case that a symmetrical matrix is given and that all eigenvalues are to be determined. For more information on the library the reader should consult Ref. [3]. There it is not only shown how the library can be applied, but also all algorithms are explained.

The program to calculate the eigenvalues reads as follows.

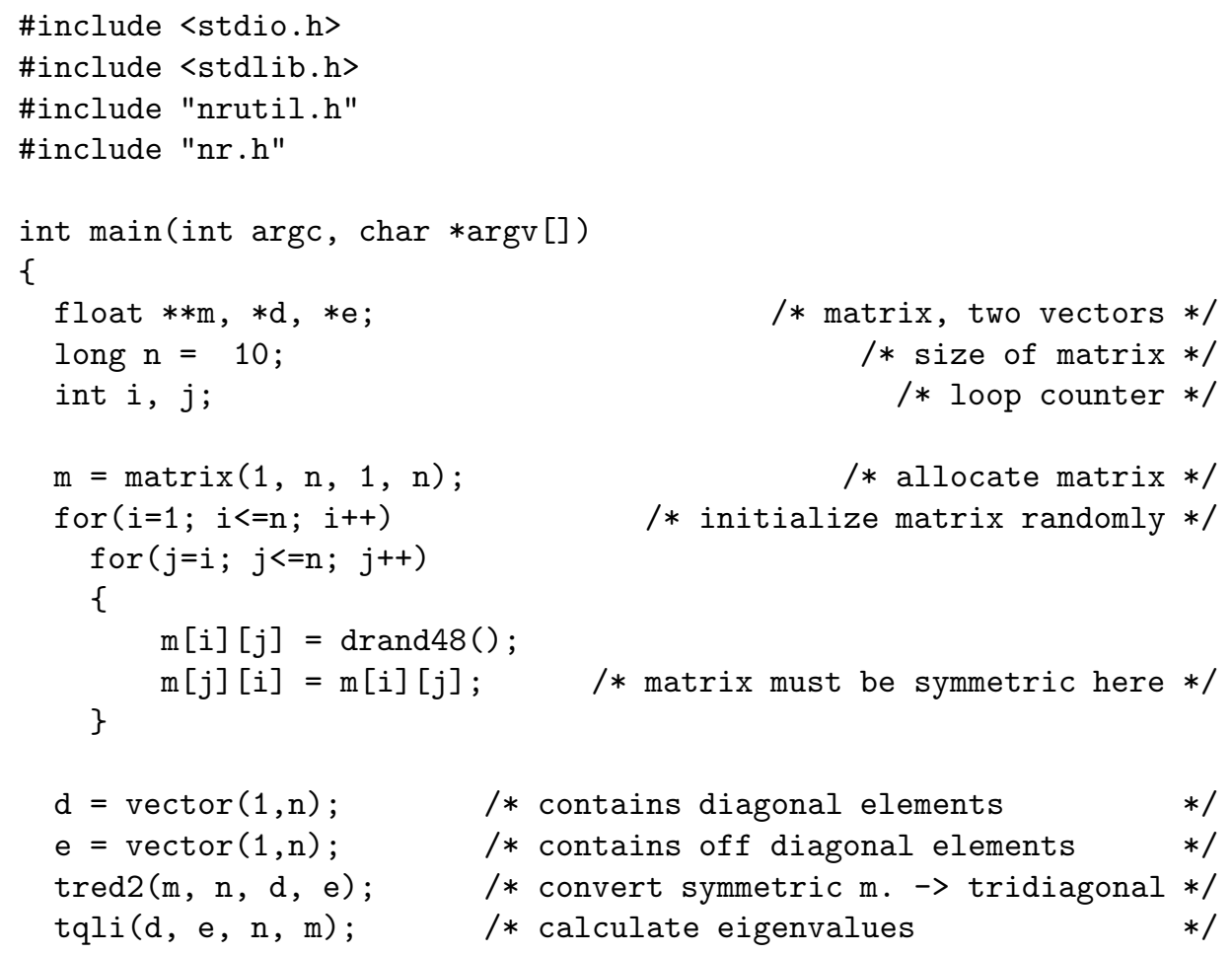




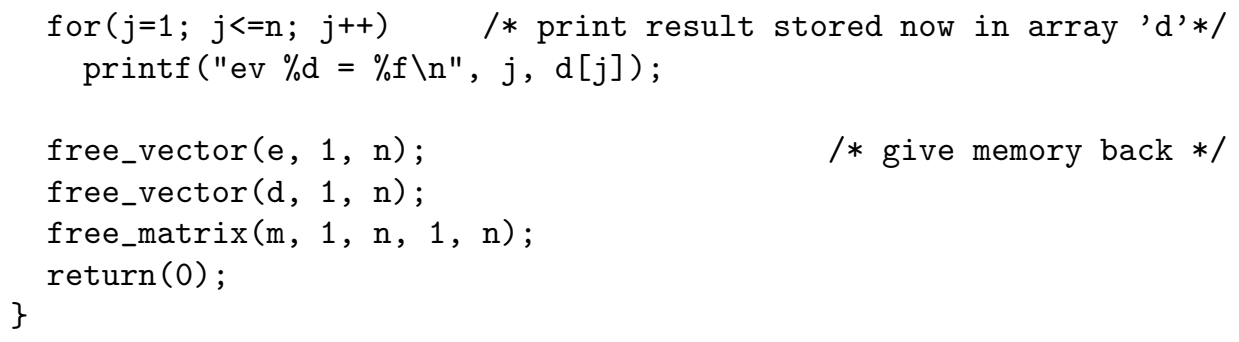

In the first part of the program, an $n \times n$ matrix is allocated via the subroutine matrix() which is provided by Numerical Recipes. It is standard to let a vector start with index 1 , while in $\mathrm{C}$ usually a vector starts with index 0 .

In the second part a matrix is initialized randomly. Since the following subroutines work only for symmetric real matrices, the matrix is initialized symmetrically. The Numerical Recipes also provide methods to diagonalize arbitrary matrices, for simplicity this special case is chosen here .

In the third part the main work is done by the Numerical Recipes subroutines tred2() and tqli(). First, the matrix is written in tridiagonal form by a Householder transformation $(\operatorname{tred} 2())$ and then the actual eigenvalues are calculated by calling tqli $(\mathrm{d}, \mathrm{e}, \mathrm{n}, \mathrm{m})$. The eigenvalues are returned in the vector $d[]$ and the eigenvectors in the matrix $m[][]$ (not used here), which is overwritten. Finally the memory allocated for the matrix and the vectors is freed again.

This small example should be sufficient to show how simply the subroutines from the Numerical Recipes can be incorporated into a program. When you have a problem of this kind you should always consult the NR library first, before starting to write code by yourself.

\subsection{LEDA}

While the Numerical Recipes are dedicated to numerical problems, the Library of Efficient Data types and Algorithms (LEDA) [4] can help a great deal in writing efficient programs in general. It is written in $\mathrm{C}++$, but it can be used by $\mathrm{C}$ style programmers as well via mixing $\mathrm{C}++$ calls to LEDA subroutines within $\mathrm{C}$ code. LEDA contains many basic and advanced data types such as:

- strings

- numbers of arbitrary precision

- one- and two-dimensional arrays

- lists and similar objects like stacks or queues

- sets

- trees

- graphs (directed and undirected, also labeled) 
- dictionaries, there you can store objects with arbitrary key words as indices

- data types for two and three dimensional geometries, like points, segments or spheres

For most data types, it is possible to create arbitrary complex structures by using templates. For example you can make lists of self defined structures or stacks of trees. The most efficient implementations known in literature so far are taken for all data structures. Usually, you can choose between different implementations, to match special requirements. For every data type, all necessary operations are included; e.g. for lists: creating, appending, splitting, printing and deleting lists as well as inserting, searching, sorting and deleting elements in a list, also iterating over all elements of a list. The major part of the library is dedicated to graphs and related algorithms. You will find for example subroutines to calculate strongly connected components, shortest paths, maximum flows, minimum cost flows and (minimum) matchings.

Here again, just a short example is given to illustrate how the library can be utilized and to show how easy LEDA can be used. A list of a self defined class Mydatatype is considered. Each element contains the data entries info and flag. In the first part of the program below, the class Mydatatype is partly defined. Please note that input and output stream operators $<</>>$ must be provided to be able to create a list of Mydatatype elements, otherwise the program will not compile. In the main part of the program a list is defined via the LEDA data type list. Elements are inserted into the list with append(). Finally an iteration over all list elements is performed using the LEDA macro forall. The program leda_test.cc reads as follows:

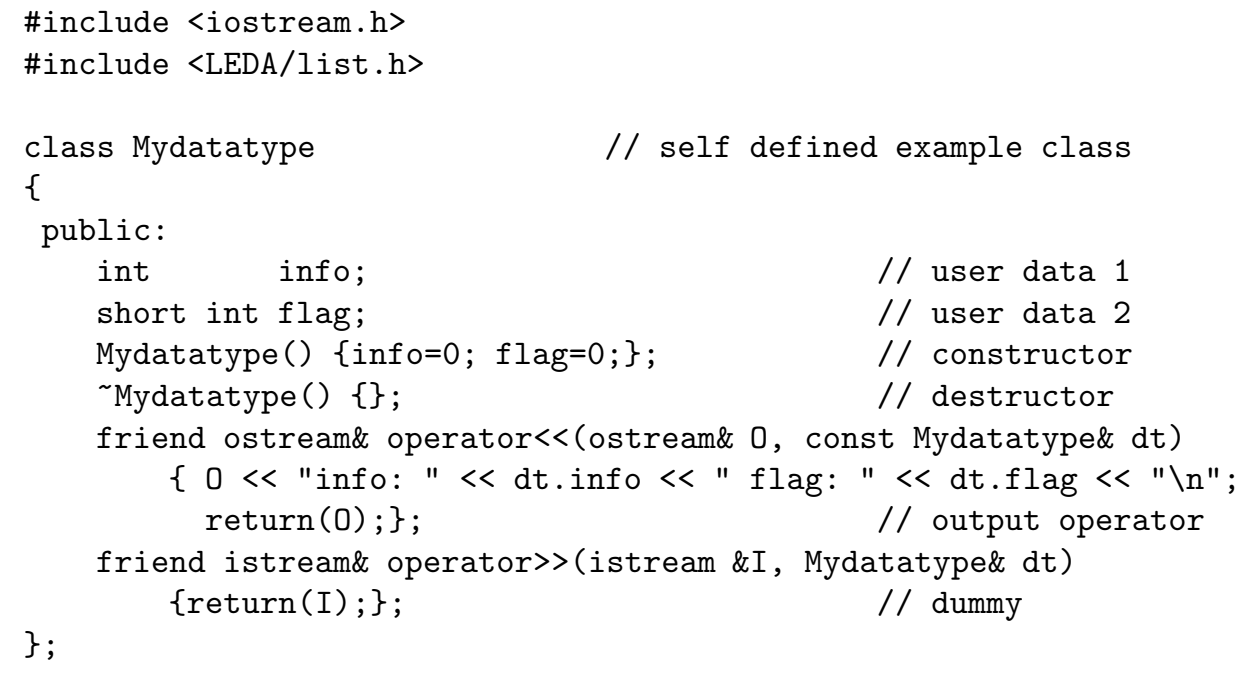




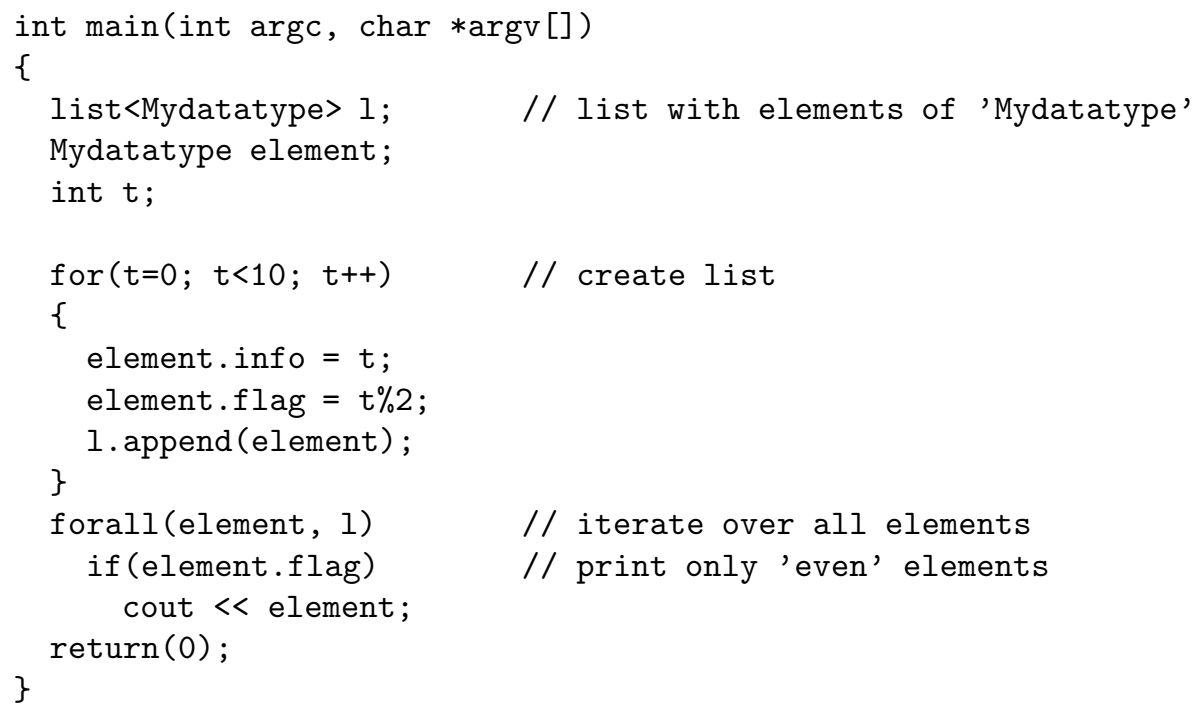

The program has to be compiled with a $\mathrm{C}++$ compiler. Depending on your system, you have to specify some compiler flags to include LEDA, please consult your systems documentation or the system administrator. The compile command may look like this:

g++ -I\$LEDAROOT/incl -L\$LEDAROOT -o leda_test leda_test.cc -lG - IL

The -I flag specifies where the compiler searches for header files like LEDA/list.h, the $-\mathrm{L}$ flag tells where the libraries $(-\mathrm{IG}-\mathrm{IL})$ are located. The environment variable LEDAROOT must point to the directory where LEDA is stored in your system.

Please note that using Numerical Recipes and LEDA together results in conflicts, since the objects vector and matrix are defined in both libraries. You can circumvent this problem by taking the source code of Numerical Recipes (here: nrutil.c, nrutil.h) and rename the subroutines matrix() and vector(), compile again and include nrutil.o directly in your program.

Here, it should be stressed: Before trying to write everything by yourself, you should check whether someone else has done it for you already. LEDA is a highly effective and very convenient tool. It will save you a lot of time and effort when you use it for your program development.

\subsection{Creating your own Libraries}

Although many useful libraries are available, sometimes you have to write some code by yourself. Over the years you will collect many subroutines, which if properly designed - can be included in other programs, in which case it is convenient to put these subroutines in a library. Then you do not have to include the object file every time you compile one of your programs. If your self-created 
library is put in a standard search path, you can access it like a system library, you even do not have to remember where the object file is stored.

To create a library you must have an object file, e.g. tasks. o, and a header file tasks. $\mathrm{h}$ where all data types and function prototypes are defined. Furthermore, to facilitate the use of the library, you should write a man page, which is not necessary for technical reasons but results in a more convenient usage of your library, particularly should other people want to benefit from it. To learn how to write a man page you should consult man man and have a look at the source code of some man pages, they are stored e.g. in /usr/man.

A library is created with the UNIX command ar. To include tasks. $\circ$ in your library libmy . a you have to enter

ar $r$ libmy.a tasks.o

In a library several object files may be collected. The option " $r$ " replaces the given object files, if they already belong to the library, otherwise they are added. If the library does not exist yet it is created. For more options, please refer to the man page of ar.

After including an object file, you have to update an internal object table of the library. This is done by

ar s libmy.a

Now you can compile a program prog.c using your library via

cc -o prog prog.c libmy.a

In case libmy . a contains several object files, it saves some typing by just writing libmy . a, furthermore you do not have to remember the names of all your object files.

To make the handling of the library more comfortable, you can create a directory, e.g. $\sim /$ lib and put your libraries there. Additionally, you should create the directory $\sim$ /include where all personal header files can be collected. Then your compile command may look like this:

cc -o prog prog.c -I\$HOME/include -L\$HOME/lib -lmy

The option -I states the search path for additional header files, the -L option tells the linker where your libraries are stored and via -lmy the library libmy.a is actually included. Please note that the prefix lib and the postfix . a are omitted with the -1 option. Finally, it should be pointed out, that the compiler command given above works in all directories, once you have set up the structure as explained. Hence, you do not have to remember directories or names of object files.

\section{Random Numbers}

For many simulations in physics, random numbers are necessary. Quite often the model itself exhibits random parameters which remain fixed throughout 
the simulation, one speaks of quenched disorder. A famous example are spin glasses. In this case one has to perform an average over different realizations of the disorder, to obtain physical quantities.

But even when the system which is treated is not random, very often random numbers are required by the algorithms, e.g. to realize a finite-temperature ensemble or when using randomized algorithms. In this section an introduction to the generation of random numbers is given. First it is explained how they can be generated at all on a computer. Then, different methods for obtaining numbers are explained, which obey a given distribution: the inversion method, the Box-Müller method and the rejection method. More comprehensive information about these and similar techniques can be found in Refs. [3, 16].

In this section it is assumed that you are familiar with the basic concepts of probability theory and statistics.

\subsection{Generating Random Numbers}

First, it should be pointed out that standard computers are deterministic machines. Thus, it is completely impossible to generate true random numbers, at least not without the help of the user. It is for example possible to measure the time interval between successive keystrokes, which is randomly distributed by nature. But they depend heavily on the current user and it is not possible to reproduce an experiment in exactly the same way. This is the reason why pseudo random numbers are usually taken. They are generated by deterministic rules, but they look like and have many of the properties of true random numbers. One would like to have a random number generator rand(), such that each possible number has the same probability of occurrence. Each time rand() is called, a new random number is returned. Additionally, if two numbers $r_{i}, r_{k}$ differ only slightly, the random numbers $r_{i+1}, r_{k+1}$ returned by the respective subsequent calls should have a low correlation.

The simplest methods to generate pseudo random numbers are linear congruential generators. They generate a sequence $I_{1}, I_{2}, \ldots$ of integer numbers between 0 and $m-1$ by a recursive recipe:

$$
I_{n+1}=\left(a I_{n}+c\right) \bmod m
$$

To generate random numbers $r$ distributed in the interval $[0,1)$ one has to divide the current random number by $m$. It is desirable to obtain equally distributed values in the interval, i.e. a uniform distribution. Below, you will see, how random numbers obeying other distributions can be generated from uniformly distributed numbers.

The real art is to choose the parameters $a, c, m$ in a way that "good" random numbers are obtained, where "good" means "with less correlations". In the past several results from simulations have been turned out to be wrong, because of the application of bad random number generators [17]. 
Example: Bad and good generators

To see what "bad generator" means, consider as an example the parameters $a=12351, c=1, m=2^{15}$ and the seed value $I_{0}=$ 1000. 10000 random numbers are generated, by dividing each of them by $m$, they are distributed in the interval $[0,1)$. In Fig. 6.1 the distribution of the random numbers is shown.

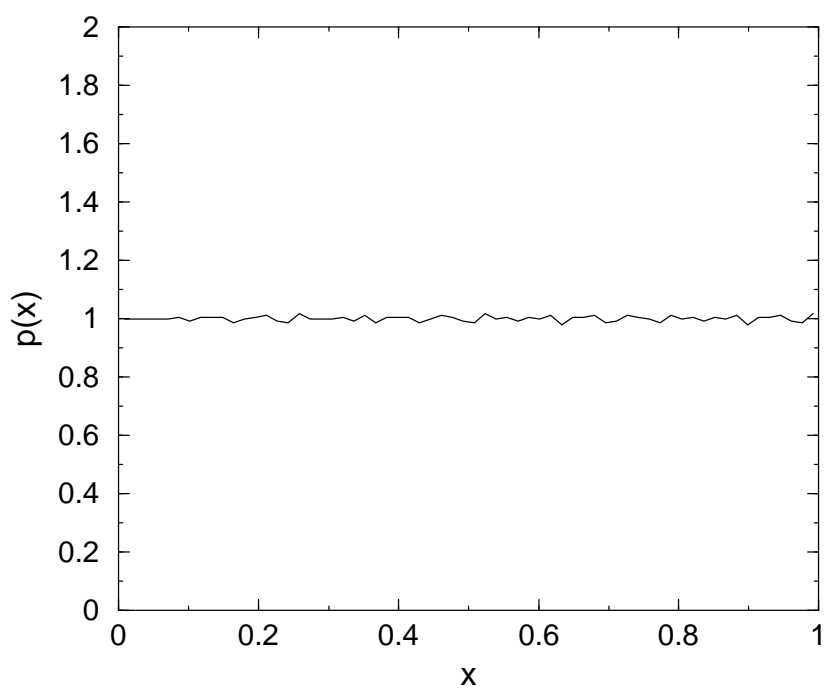

Figure 2: Distribution of random numbers in the interval $[0,1)$. They are generated using a linear congruential generator with the parameters $a=12351, c=1, m=2^{15}$.

The distribution looks rather flat, but by taking a closer look some regularities can be observed. These regularities can be studied by recording $k$-tuples of $k$ successive random numbers $\left(x_{i}, x_{i+1}, \ldots, x_{i+k-1}\right)$. A good random number generator, exhibiting no correlations, would fill up the $k$-dimensional space uniformly. Unfortunately, for linear congruential generators, instead the points lie on $(k-1)$-dimensional planes. It can be shown that there are at most of the order $m^{1 / k}$ such planes. A bad generator has much fever planes. This is the case for the example studied above, see top part of Fig. 6.1

The result for $a=123450$ is even worse, only 15 different "random" numbers are generated (with seed 1000), then the iteration reaches a fixed point (not shown in a figure).

If instead $a=12349$ is chosen, the two-point correlations look like that shown in the bottom half of Fig. 6.1. Obviously, the behavior is 

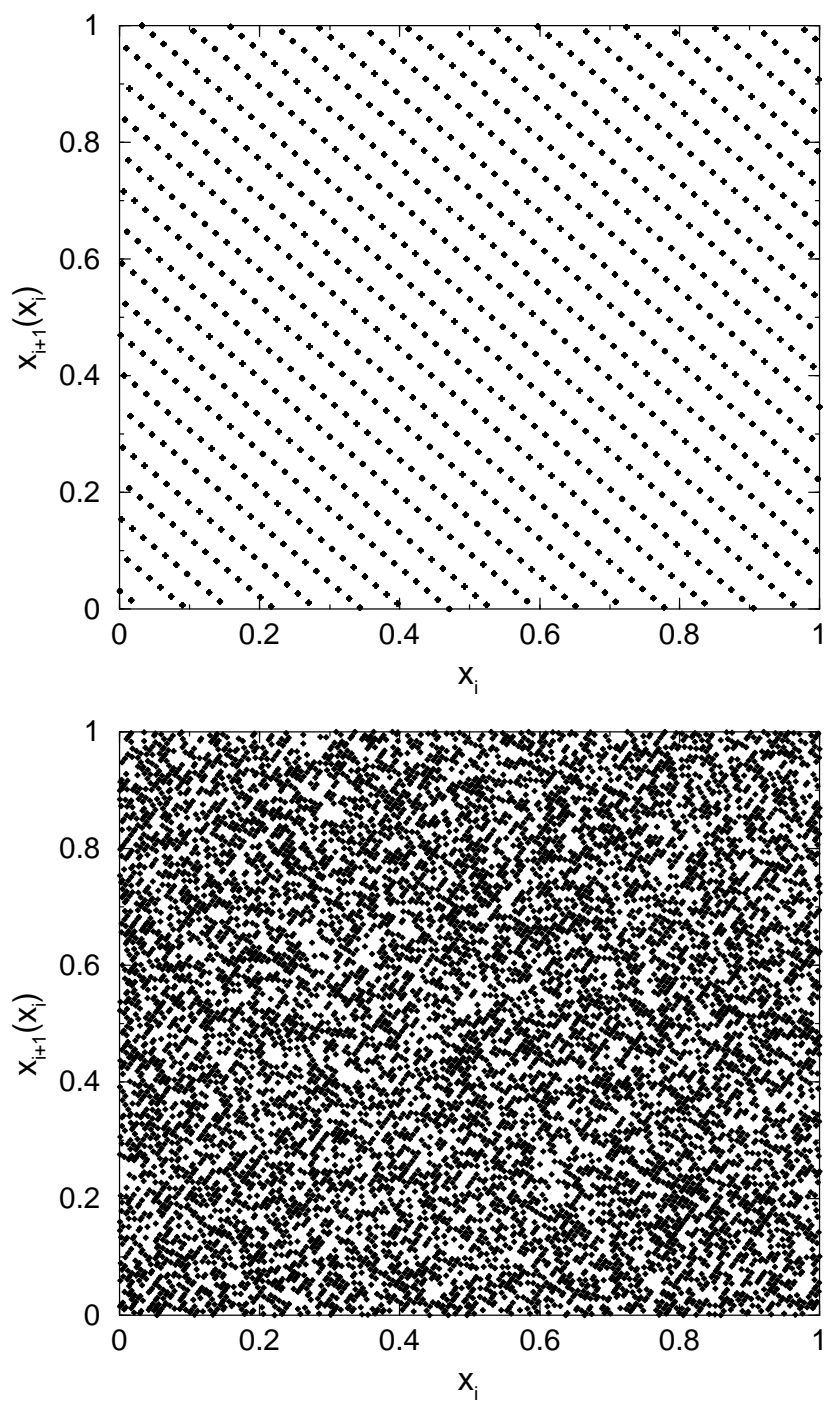

Figure 3: Two point correlations $x_{i+1}\left(x_{i}\right)$ between successive random numbers $x_{i}, x_{i+1}$. The top case is generated using a linear congruential generator with the parameters $a=12351, c=1, m=2^{15}$, the bottom case has instead $a=12349$.

much more irregular, but poor correlations may become visible for higher $k$-tuples. 
A generator which has passed several theoretical test is $a=7^{5}=16807, m=$ $2^{31}-1, c=0$. When implementing this generator you have to be careful, because during the calculation numbers are generated which do not fit into 32 bit. A clever implementation is presented in Ref. [3]. Finally, it should be stressed that this generator, like all linear congruential generators, has the loworder bits much less random than the high-order bits. For that reason, when you want to generate integer numbers in an interval $[1, N]$, you should use

$$
\mathrm{r}=1+(\text { int }) \quad\left(\mathrm{N} *\left(\mathrm{I} \_\mathrm{n}\right) / \mathrm{m}\right) \text {; }
$$

instead of using the modulo operation as with $r=1+\left(I \_n \% N\right)$;

So far it has been shown how random numbers can be generated which are distributed uniformly in the interval $[0,1)$. In general, one is interested in obtaining random numbers which are distributed according to a given probability distribution with density $p(z)$. In the next sections, several techniques performing this task for continuous probability distributions are presented.

In case of discrete distributions, one has to create a table of the possible outcomes with their probabilities $p_{i}$. To draw a number, one has to draw a random number $u$ which is uniformly distributed in $[0,1)$ and take the entry $j$ of the table such that the sum $\sum_{i=1}^{j} p_{i}$ of the preceding probabilities is larger than $u$, but $\sum_{i=1}^{j-1} p_{i}<u$. In the following, we concentrate on techniques for generating continuous random variables.

\subsection{Inversion Method}

Given is a random number generator drand() which is assumed to generate random numbers $U$ which are distributed uniformly in $[0,1)$. The aim is to generate random numbers $Z$ with probability density $p(z)$. The corresponding distribution function is

$$
P(z) \equiv \operatorname{Prob}(Z \leq z) \equiv \int_{-\infty}^{z} d z^{\prime} p\left(z^{\prime}\right)
$$

The target is to find a function $g(X)$, such that after the transformation $Z=$ $g(U)$, the values of $Z$ are distributed according to (2). It is assumed that $g$ can be inverted and is strongly monotonically increasing, then one obtains

$$
P(z)=\operatorname{Prob}(Z \leq z)=\operatorname{Prob}(g(U) \leq z)=\operatorname{Prob}\left(U \leq g^{-1}(z)\right)
$$

Since the distribution function $F(u)=\operatorname{Prob}(U \leq u)$ for a uniformly distributed variable is just $F(u)=u(u \in[0,1])$, one obtains $P(z)=g^{-1}(z)$. Thus, one just has to choose $g(z)=P^{-1}(z)$ for the transformation function, in order to obtain random numbers, which are distributed according the probability distribution $P(z)$. Of course, this only works if $P$ can be inverted. 
Example: Exponential distribution

Let us consider the exponential distribution with parameter $\lambda$, with probability density

$$
p(z)=\lambda \exp (-\lambda z)
$$

and distribution function $P(z)=1-\exp (-\lambda z)$. Therefore, one can obtain exponentially distributed random numbers $Z$, by generating uniform distributed random numbers $U$ and choosing $Z=-\ln (1-$ $U) / \lambda$.

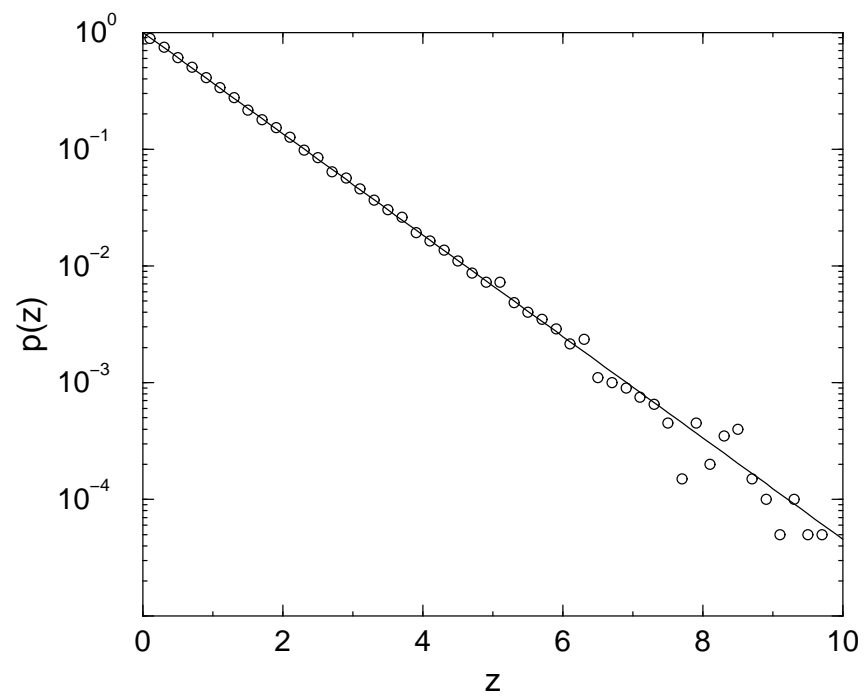

Figure 4: Histogram of random numbers generated according to an exponential distribution $(\lambda=1)$ compared with the probability density (straight line) in a logarithmic plot.

In Fig. 6.2 a histogram for $10^{5}$ random numbers generated in this way and the exponential probability function for $\lambda=1$ are shown with a logarithmically scaled $y$-axis. Only for larger values are deviations visible. They are due to statistical fluctuations since $p(z)$ is very small there.

For completeness, this example is finished by mentioning that by summing $n$ independent exponentially distributed random numbers, the result is gamma distributed [16]. 


\subsection{Rejection Method}

As mentioned above, the inversion method works only when the distribution function $P$ can be inverted. For distributions not fulfilling this condition, sometimes this problem can be overcome by drawing several random numbers and combining them in a clever way, see e.g. the next subsection.

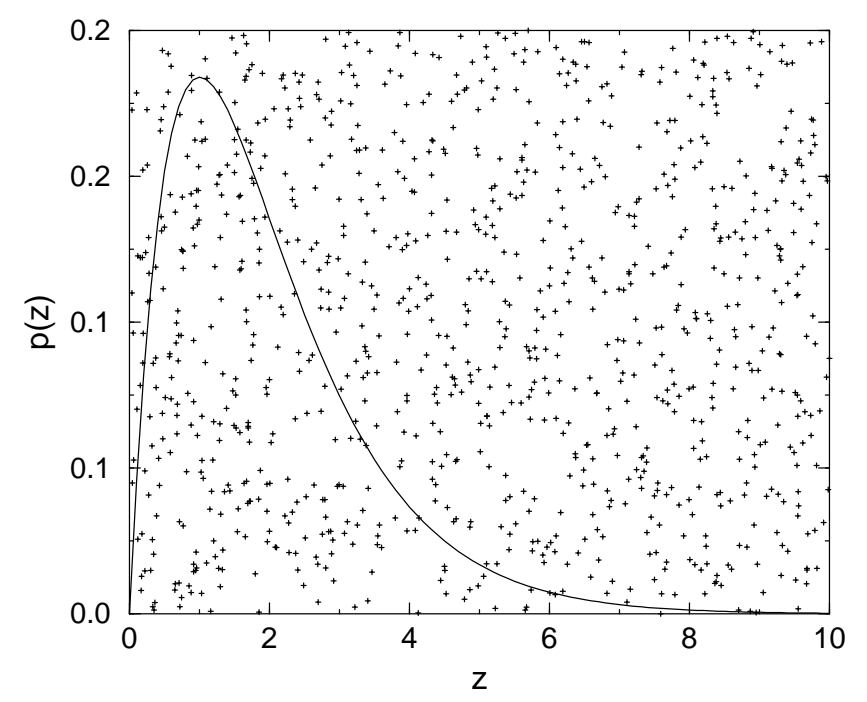

Figure 5: The rejection method: points $(x, y)$ are scattered uniformly over a bounded rectangle. The probability that $y \leq p(x)$ is proportional to $p(x)$.

The rejection method, which is presented in this section, works for random variables where the probability distribution $p(z)$ fits into a box $\left[x_{0}, x_{1}\right) \times\left[0, z_{\max }\right)$, i.e. $p(z)=0$ for $z \notin\left[x_{0}, x_{1}\right]$ and $p(z) \leq z_{\max }$. The basic idea of generating a random number distributed according to $p(z)$ is to generate random pairs $(x, y)$, which are distributed uniformly in $\left[x_{0}, x_{1}\right] \times\left[0, z_{\max }\right]$ and accept only those values $x$ where $y \leq p(x)$ holds, i.e. the pairs which are located below $p(x)$, see Fig. 5 . Therefore, the probability that $x$ is drawn is proportional to $p(x)$, as desired. The algorithm for the rejection method is: 


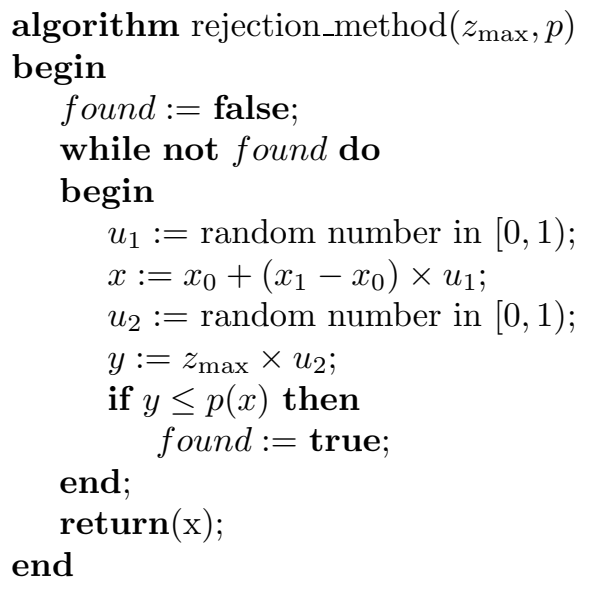

The rejection method always works if the probability density is boxed, but it has the drawback that more random numbers have to be generated than can be used.

In case neither the distribution function can be inverted nor the probability fits into a box, special methods have to be applied. As an example a method for generating random numbers distributed according to a Gaussian distribution is considered. Other methods and examples of how different techniques can be combined, are collected in Ref. [16].

\subsection{The Gaussian Distribution}

The probability density for the Gaussian distribution with mean $m$ and width $\sigma$ is (see also Fig. 6)

$$
p_{G}(z)=\frac{1}{\sqrt{2 \pi} \sigma} \exp \left(\frac{(z-m)^{2}}{2 \sigma^{2}}\right)
$$

It is, apart from uniform distributions, the most common distribution being applied in simulations.

Here, the case of a normal distribution $(m=0, \sigma=1)$ is considered. If you want to realize the general case, you have to draw a normally distributed number $z$ and then use $\sigma z+m$ which is distributed as desired.

Since the normal distribution extends over an infinite interval and cannot be inverted, the methods from above are not applicable. The simplest technique to generate random numbers distributed according to a normal distribution makes use of the central limit theorem. It tells us that any sum of $N$ independently distributed random variables $u_{i}$ (with mean $m$ and variance $v$ ) will converge to a Gaussian distribution with mean $N m$ and variance $N v$. If again $u_{i}$ is taken take to be uniformly distributed in $[0,1$ ) (which has mean $m=0.5$ and variance $v=1 / 12$ ), one can choose $N=12$ and $Z=\sum_{i=1}^{12} u_{i}-6$ will be distributed approximately normally. The drawback of this method is that 12 


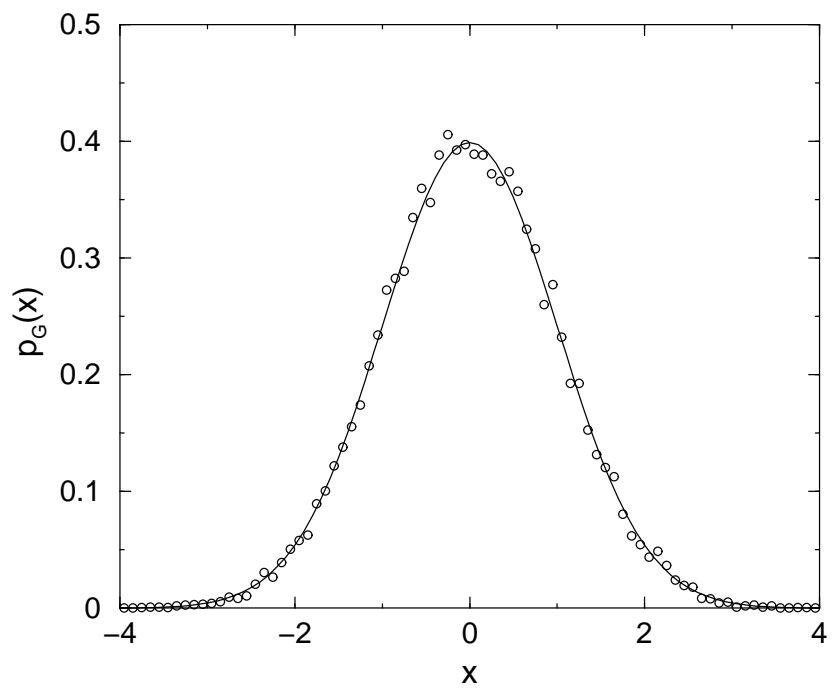

Figure 6: Gaussian distribution with zero mean and unit width. The circles represent a histogram obtained from $10^{4}$ values drawn with the Box-Müller method.

random numbers are needed to generate one final random number and that values larger than 6 never appear.

In contrast to this technique the Box-Müller method is exact. You need two uniformly in $[0,1)$ distributed random variables $U_{1}, U_{2}$ to generate two independent normal variables $N_{1}, N_{2}$. This can be achieved by setting

$$
\begin{aligned}
& N_{1}=\sqrt{-2 \log \left(1-u_{1}\right)} \cos \left(2 \pi u_{2}\right) \\
& N_{2}=\sqrt{-2 \log \left(1-u_{1}\right)} \sin \left(2 \pi u_{2}\right)
\end{aligned}
$$

A proof that $N_{1}$ and $N_{2}$ are indeed distributed according to (5) can be found in Refs. [3, 16], where also other methods for generating Gaussian random numbers, some even more efficient, are explained. A method which is based on the simulation of particles in a box is explained in Ref. [18]. In Fig. 6 a histogram of $10^{4}$ random numbers drawn with the Box-Müller method is shown.

\section{Tools for Testing}

In Sec. 1 the importance of thorough testing has already been stressed. Here three useful tools are presented which significantly assist in facilitating the debugging process. Please note again that the tools run under UNIX/Linux operating systems. Similar programs are available for other operating systems as well. The tools covered here are $g d b$, a source-code debugger, $d d d$, a graphic 
front-end to gdb, and checkergcc, which finds bugs resulting from bad memory management.

\section{$7.1 \quad g d b$}

The $g d b$ gnu debugger tool is a source code debugger. Its main purpose is that you can watch the execution of your code. You can stop the program at arbitrarily chosen points by setting breakpoints at lines or subroutines in the source code, inspect variables/data structures, change them and let the program continue (e.g. line by line). Here some examples for the most basic operations are given, detailed instructions can be obtained within the program via the help command.

As an example of how to debug, please consider the following little program gdbtest.c:

\#include <stdio.h>

\#include <stdlib.h>

int main(int argc, char $* \operatorname{argv}[]$ )

\{

int $t, * a r r a y$, sum $=0$;

array $=($ int $*)$ malloc $(100 *$ sizeof (int $))$;

for $(t=0 ; t<100 ; t++)$

array $[\mathrm{t}]=\mathrm{t}$;

for $(t=0 ; t<100 ; t++)$

sum $+=\operatorname{array}[t]$;

printf ("sum $=\% d \backslash n "$, sum $)$;

free (array);

return $(0)$;

\}

When compiling the code you have to include the option -g to allow debugging:

cc -o gdbtest -g gdbtest.c

The debugger is invoked using gdb $<$ programname $>$, i.e.

gdb gdbtest

Now you can enter commands, e.g. list the source code of the program via the list command, it is sufficient to enter just 1. By default always ten lines at the current position are printed. Therefore, at the beginning the first ten lines are shown (the first line shows the input, the other lines state the answer of the debugger) 
$(\mathrm{gdb}) 1$

1 \#include <stdio.h>

2 \#include <stdlib.h>

4 int main(int argc, char *argv[])

5\{

6

7

$8 \quad$ array $=$ (int $*) \operatorname{malloc}(100 * \operatorname{sizeof}($ int $))$;

9 for $(t=0 ; t<100 ; t++)$

$10 \quad \operatorname{array}[\mathrm{t}]=\mathrm{t}$;

When entering the command again the next ten lines are listed. Furthermore, you can refer to program lines of the code in the form list $\langle$ from $\rangle,\langle$ to $\rangle$ or to subroutines by typing list <name of subroutine $>$. More information can be obtained by typing help list.

To let the execution stop at a specific line one can use the break command (abbreviation $\mathrm{b}$ ). To stop the program before line 11 is executed, one enters

(gdb) b 11

Breakpoint 1 at 0x80484b0: file gdbtest.c, line 11.

Breakpoints can be removed via the delete command. All current breakpoints are displayed by entering info break.

To start the execution of the program, one enters run or just $r$. As requested before, the program will stop at line 11:

(gdb) $r$

Starting program: gdbtest

Breakpoint 1, main ( $\operatorname{argc}=1, \operatorname{argv}=0 x b f f f f 384)$ at gdbtest.c $: 11$

11 for $(t=0 ; t<100 ; t++)$

Now you can inspect for example the content of variables via the print command:

(gdb) p array

$\$ 1=($ int $*)$ 0x8049680

(gdb) p array [99]

$\$ 2=99$

To display the content of a variable permanently, the display command is available. You can change the content of variables via the set command

(gdb) set array [99] $=98$

You can continue the program at each stage by typing next, then just the next source-code line is executed: 
(gdb) $\mathrm{n}$

Subroutines are regarded as one source-code line as well. If you want to debug the subroutine in a step-wise manner as well you have to enter the step command. By entering continue, the execution is continued until the next breakpoint, a severe error, or the end of the program is reached, please note the the output of the program appears in the $g d b$ window as well:

(gdb) c

Continuing.

sum $=4949$

Program exited normally.

As you can see, the final value (4949) the program prints is affected by the change of the variable array [99].

The above given commands are sufficient for most of the standard debugging tasks. For more specialized cases $g d b$ offers many other commands, please have a look at the documentation [5].

\section{$7.2 \quad d d d$}

Some users may find graphical user interfaces more convenient. For this reason there exists a graphical front-end to the gdb, the data display debugger (ddd). On UNIX operating systems it is just invoked by typing ddd (see also man page for options). Then a nice windows pops up, see Fig. 7. The lower part of the window is an ordinary $g d b$ interface, several other windows are available. By typing file <program $>$ you can load a program into the debugger. Then the source code is shown in the main window of the debugger. All $g d b$ commands are available, the most important ones can be entered via menus or buttons using the mouse. For example to set a breakpoint it is sufficient to place the cursor in a source-code line in the main ddd window and click on the break button. A good feature is that the content of a variable is shown when moving the mouse onto it. For more details, please consult the online help of ddd.

\section{3 checkergcc}

Most program bugs are revealed by systematically running the program and cross-checking with the expected results. But other errors seem to appear in a rather irregular and unpredictable fashion. Sometimes a program runs without a problem, in other cases it crashes with a Segmentation fault at rather puzzling locations in the code. Very often a bad memory management is the cause of such a behavior. Writing beyond the boundaries of an array, reading uninitialized memory locations or addressing data which has been freed already are the most common bugs of this class. Since the operating system organizes the memory in a different way each time a program is run, it is rather unpredictable whether 


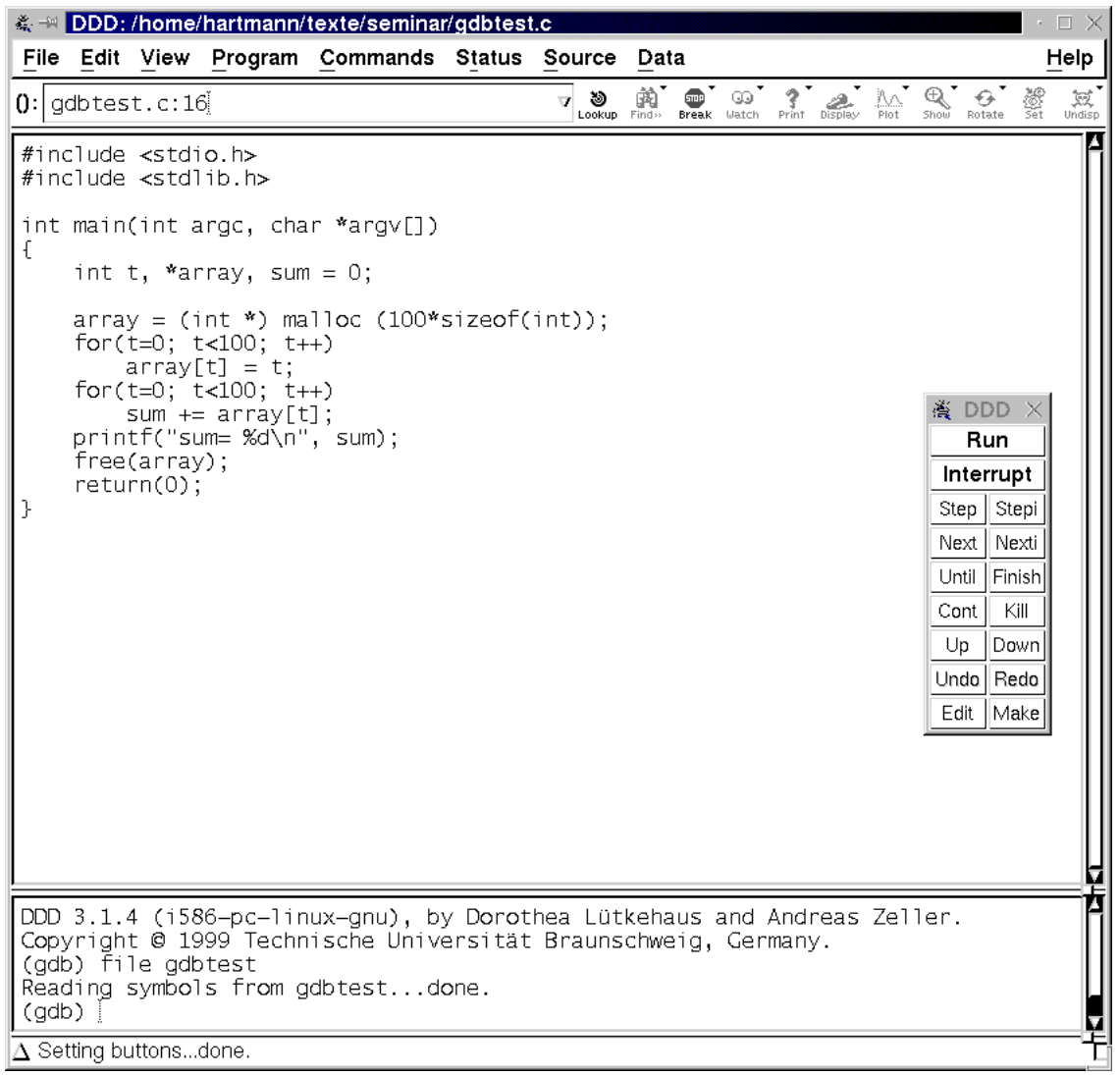

Figure 7: The data display debugger (ddd). In the main window the source code is shown. Commands can be invoked via a mouse or by entering them into the lower part of the window.

these errors become apparent or not. Furthermore it is very hard to track them down, because the effect of such errors most of the time becomes visible at positions different from where the error has occurred.

As an example, the case where it is written beyond the boundary of an array is considered. If in the heap, where all dynamically allocated memory is taken from, at the location behind the array another variable is stored, it will be overwritten in this case. Hence, the error becomes visible the next time the other variable is read. On the other hand, if the memory block behind the array is not used, the program may run that time without any problems. Unfortunately, the programmer is not able to influence the memory management directly.

To detect such types of nasty bugs, one can take advantage of several tools. A list of free and commercial tools can be found in Ref. [19]. Here checkergcc is considered, which is a very convenient tool and freely available. It works under 
UNIX and is included by compiling everything with checkergcc instead of cc or gcc. Unfortunately, the current version does not have full support for $\mathrm{C}++$, but you should try it on your own project. The checkergcc compiler replaces all memory allocations/deallocations and accesses by its own routines. Any access to non-authorized memory locations is reported, regardless of the positions of other variables in the memory area (heap).

As an example, the program from Sec. 7.1 is considered, which is slightly modified; the memory block allocated for the array is now slightly too short (length 99 instead of 100):

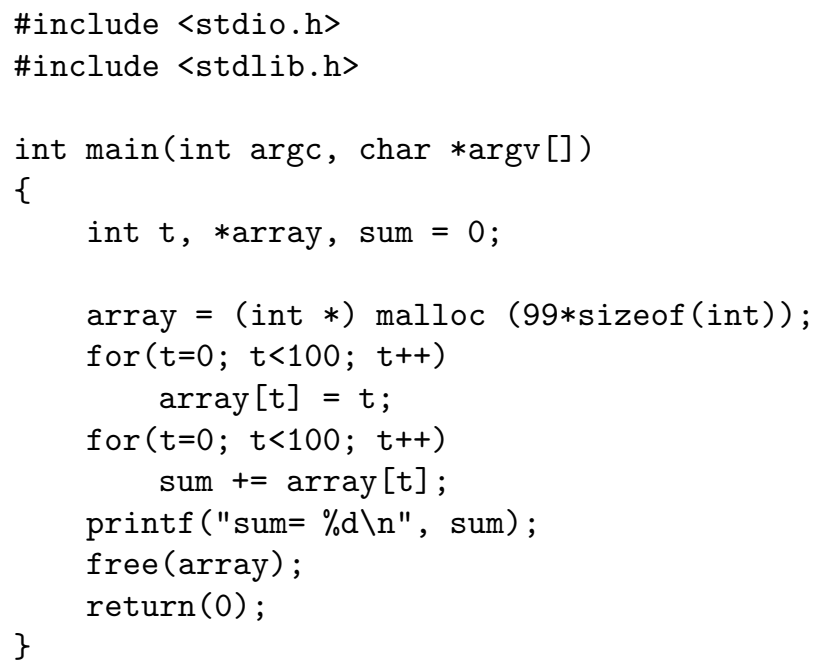

The program is compiled via

checkergcc -o gdbtest -g gdbtest.c

Starting the program produces the following output, the program terminates normally:

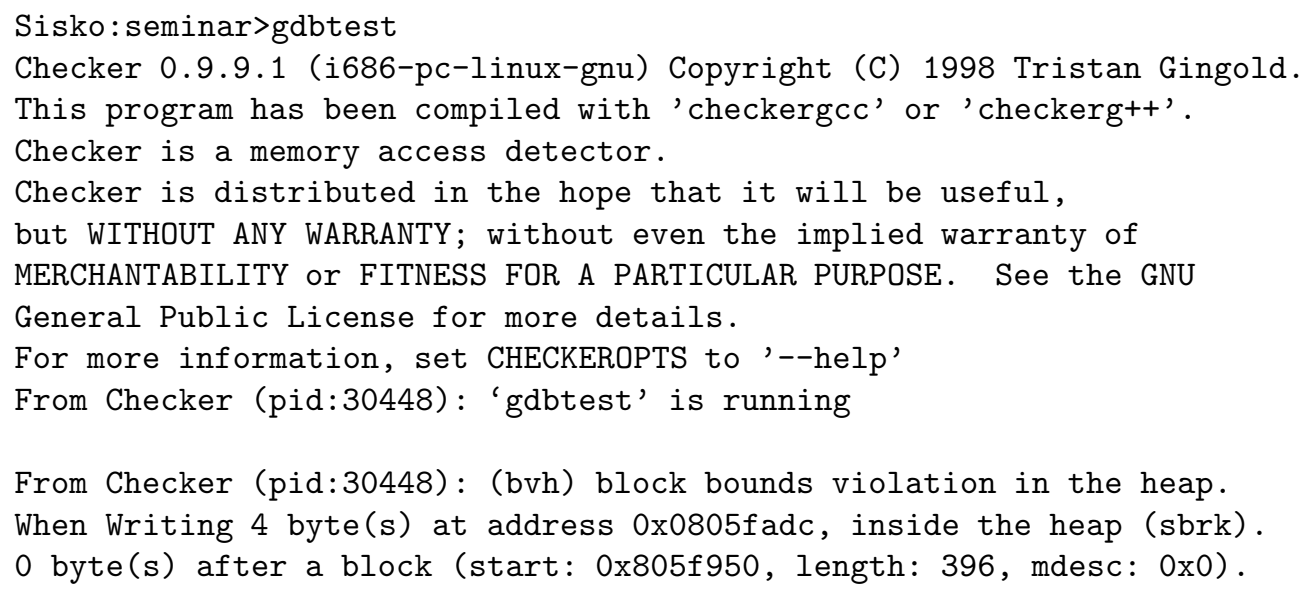


The block was allocated from:

pc=0x080554f 9 in chkr_malloc at stubs-malloc.c:57

pc $=0 \times 08048863$ in main at gdbtest. $c: 8$

$\mathrm{pc}=0 \mathrm{x} 080555 \mathrm{a} 7$ in this_main at stubs-main.c:13

pc $=0 \times 40031 \mathrm{c} 7 \mathrm{e}$ in _-_divdi3 at stubs/end-stubs.c:7

pc $=0 \times 08048668$ in *unknown* at *unknown*:0

Stack frames are:

pc $=0 \times 080489 \mathrm{c} 3$ in main at gdbtest. $c: 10$

pc $=0 \times 080555 \mathrm{a} 7$ in this_main at stubs-main.c:13

pc $=0 \times 40031 \mathrm{c} 7 \mathrm{e}$ in _-_divdi3 at stubs/end-stubs.c:7

pc $=0 \times 08048668$ in *unknown* at *unknown*:0

From Checker (pid:30448): (bvh) block bounds violation in the heap.

When Reading 4 byte(s) at address 0x0805fadc, inside the heap (sbrk).

0 byte(s) after a block (start: 0x805f950, length: 396, mdesc: 0x0).

The block was allocated from:

pc $=0 \times 00000063$ in $*$ unknown $*$ at $*$ unknown $*: 0$

pc $=0 \times 08048863$ in main at gdbtest. $c: 8$

pc $=0 \times 080555 \mathrm{a} 7$ in this_main at stubs-main.c:13

pc $=0 \times 40031 \mathrm{c} 7 \mathrm{e}$ in _-_divdi3 at stubs/end-stubs.c:7

pc $=0 \times 08048668$ in *unknown* at *unknown*:0

Stack frames are:

pc $=0 \times 08048 c 55$ in main at gdbtest.c: 12

pc $=0 x 080555 a 7$ in this_main at stubs-main.c:13

pc $=0 \times 40031 \mathrm{c} 7 \mathrm{e}$ in _-_divdi3 at stubs/end-stubs.c:7

pc $=0 \times 08048668$ in *unknown $*$ at *unknown*:0

Two errors are reported, each message starts with "From checker". Both errors consist of accesses to an array beyond the border (block bound violation). For each error both the location in the source code where the memory has been allocated and the location where the error occurred (Stack frames) are given. In both cases the error is concerned with what was allocated at line 8 (pc=0x08048863 in main at gdbtest.c:8). The bug appeared during the loops over the array, when the array is initialized (line 10) and read out (line $12)$.

Other common types of errors are memory leaks. They appear when a previously used block of memory has been forgotten to be freed again. Assume that this happens in a subroutine which is called frequently in a program. You can imagine that you will quickly run out of memory. Memory leaks are not detected using checkergcc by default. This kind of test can be turned on by setting a special environment variable CHECKEROPTS, which controls the behavior of checkergcc. To enable checking for memory leaks at the end of the execution, one has to set

export CHECKEROPTS $="-\mathrm{D}=$ end"

Let us assume that the bug from above is removed and instead the free (array); command at the end of the program is omitted. After compiling with checkergcc, running the program results in: 


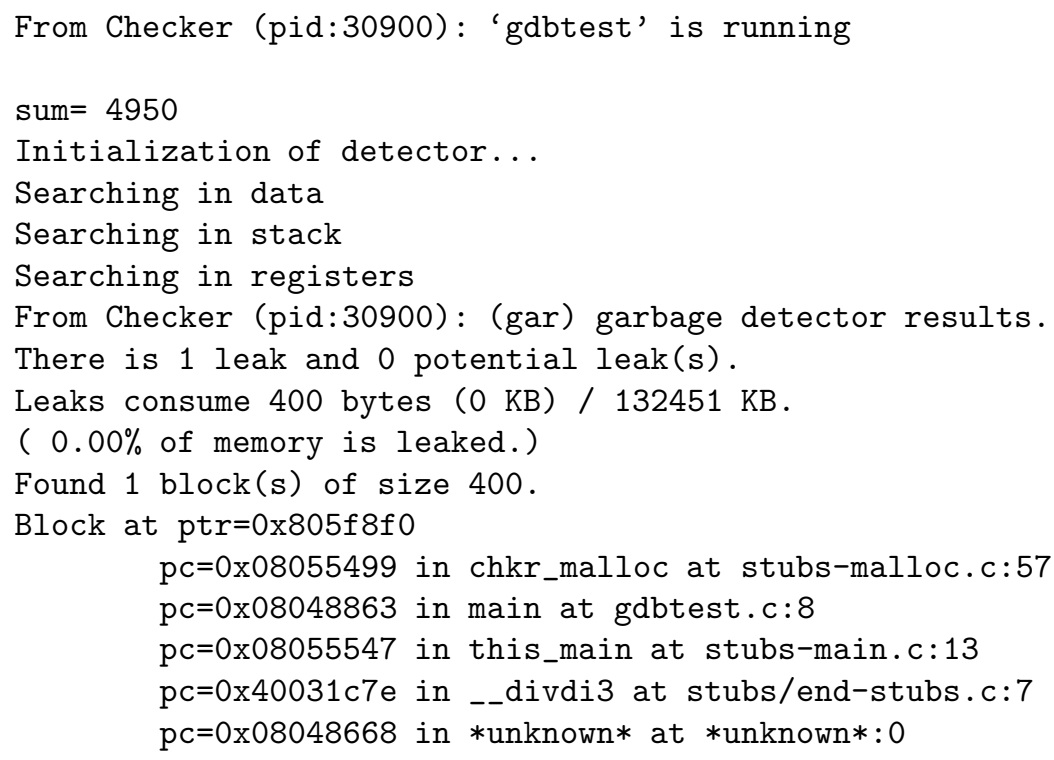

Obviously, the memory leak has been found. Further information on the various features of checkergcc can be found in Ref. [20]. A last hint: you should always test a program with a memory checker, even if everything seems to be fine.

\section{Evaluating Data}

To analyze and plot data, several commercial and non-commercial programs are available. Here three free programs are discussed, gnuplot, xmgr and fsscale. Gnuplot is small, fast, allows two- and three-dimensional curves to be generated and to fit arbitrary functions to the data. On the other hand $x m g r$ is more flexible and produces better output. It is recommended that gnuplot is used for viewing and fitting data online, while $x m g r$ is to be preferred for producing figures to be shown in talks or publications. The program fsscale has a special purpose. It is very convenient for performing finite-size scaling plots.

First, gnuplot and xmgr are introduced with respect to drawing figures. In the next subsection, data fitting is covered. Finally, it is shown how finite-size scaling plots can be created. In all three cases only very small examples can be presented. They should serve just as a motivation to study the documentation, then you will learn about the manifold potential the programs offer.

\subsection{Data Plotting}

The program gnuplot is invoked by entering gnuplot in a shell, for a complete manual see Ref. [13]. As always, our examples refer to a UNIX window system like X11, but the program is available for almost all operating systems. After startup, the prompt (e.g. gnuplot $>$ ) appears and the user can enter commands in textual form, results are shown in windows or are written into files. Before 
giving an example, it should be pointed out that gnuplot scripts can be generated by simply writing the commands into a file, e.g. command.gp, and calling gnuplot command.gp.

The typical case is that you have a data file of $x-y$ data and you want to plot the figure. Your file might look like this, it is the ground-state energy of a three-dimensional $\pm J$ spin glass as a function of the linear system size $L$. The filename is sg_e0_L.dat. The first column contains the $L$ values, the second the energy values and the third the standard error of the energy, please note that lines starting with "\#” are comment lines which are ignored on reading:

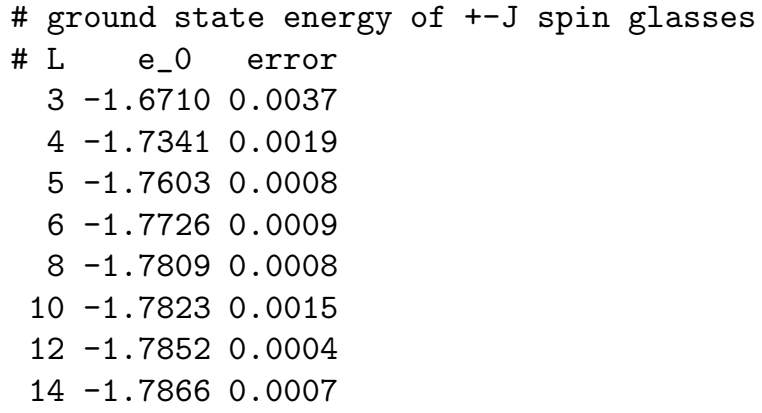

To plot the data enter

gnuplot> plot "sg_e0_L.dat" with yerrorbars

which can be abbreviated as p "sg_e0_L.dat" w e. Please do not forget the quotation marks around the file name. Next, a window pops up, showing the result, see Fig. 8.

For the plot command many options and styles are available, e.g. with lines produces lines instead of symbols. It is possible to read files with multi columns via the using option, e.g.

gnuplot> plot "test.dat" using 1:4:5 w e

displays the fourth column as a function of the first, with error bars given by the 5 th column. Among other options, it is possible to redirect the output, for example to an encapsulated postscript file (by setting set terminal postscript and redirecting the output set output "test.eps"). Also several files can be combined into one figure. You can set axis labels of the figure by typing e.g. set xlabel "L", which becomes active when the next plot command is executed. Online help on the plot command and its manifold options is available via entering help plot. Also three-dimensional plotting is possible using the splot command (enter help splot to obtain more information). For a general introduction you can type just help. Since gnuplot commands can be entered very quickly, you should use it for online viewing data and fitting (see Sec. 8.2).

The $x m g r$ (x motiv graphic) program is much more powerful than gnuplot and produces nicer output, commands are issued by clicking on menus and buttons. 


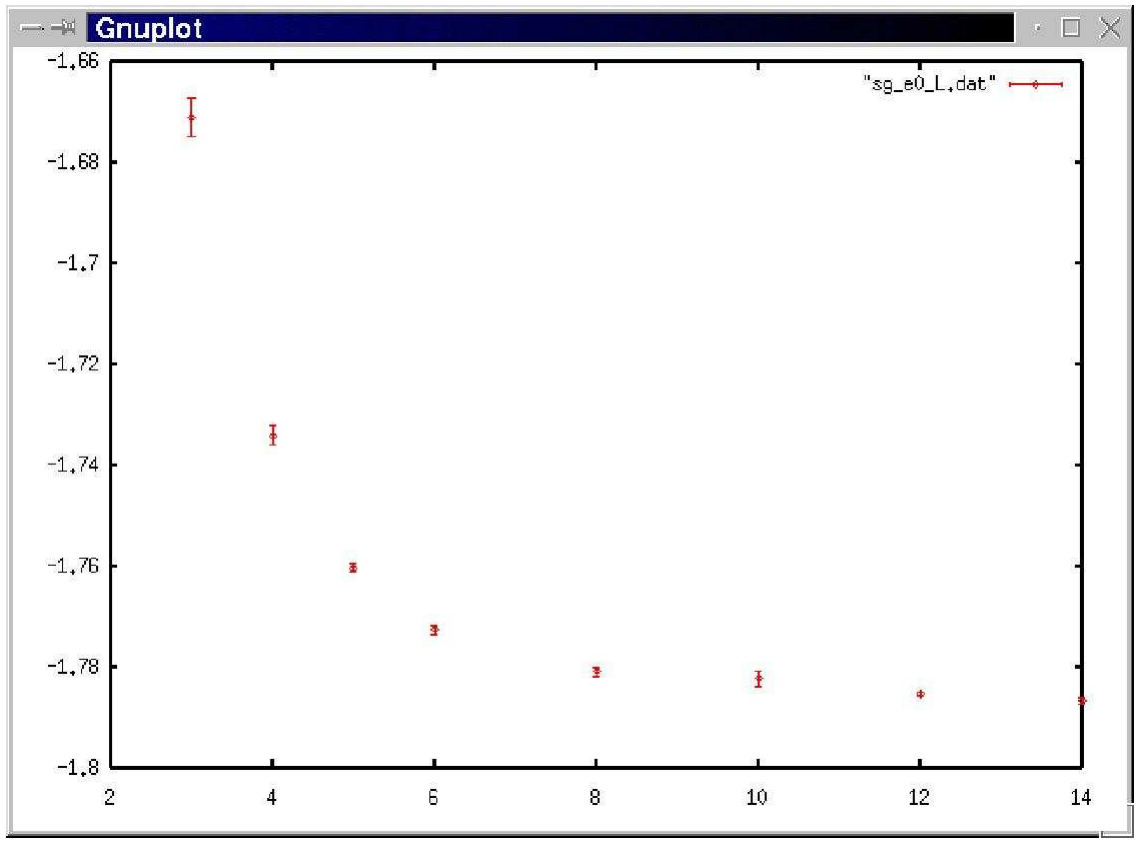

Figure 8: Gnuplot window showing the result of a plot command.

On the other hand its handling is a little bit slower and the program has the tendency to fill your screen with windows. To create a similar plot to that above, you have to go (after staring it by typing xmgr into a shell) to the files menu and choose the read submenu and the sets subsubmenu. Then a file selection window will pop up and you can choose the data file to be loaded. The situation is shown in Fig. 9.

The xmgr program offers almost every feature you can imagine for two-dimensional data plots, including multiple plots, fits, many styles for lines, symbols, bar charts etc. Also you can create manifold types of labels or legends and it is possible to add elements like strings, lines or other geometrical objects in the plot. For more information, please consult the online help.

\subsection{Curve Fitting}

Both programs presented above, gnuplot and xmgr, offer fitting of arbitrary functions. It is advisable to use gnuplot, since it offers a higher flexibility for that purpose and gives you more information useful to estimate the quality of a fit.

As an example, let us suppose that you want to fit an algebraic function of the form $f(L)=e_{\infty}+a L^{b}$ to the data set of the file sg_e0_L.dat shown above. First, you have to define the function and supply some roughly (non-zero) esti- 


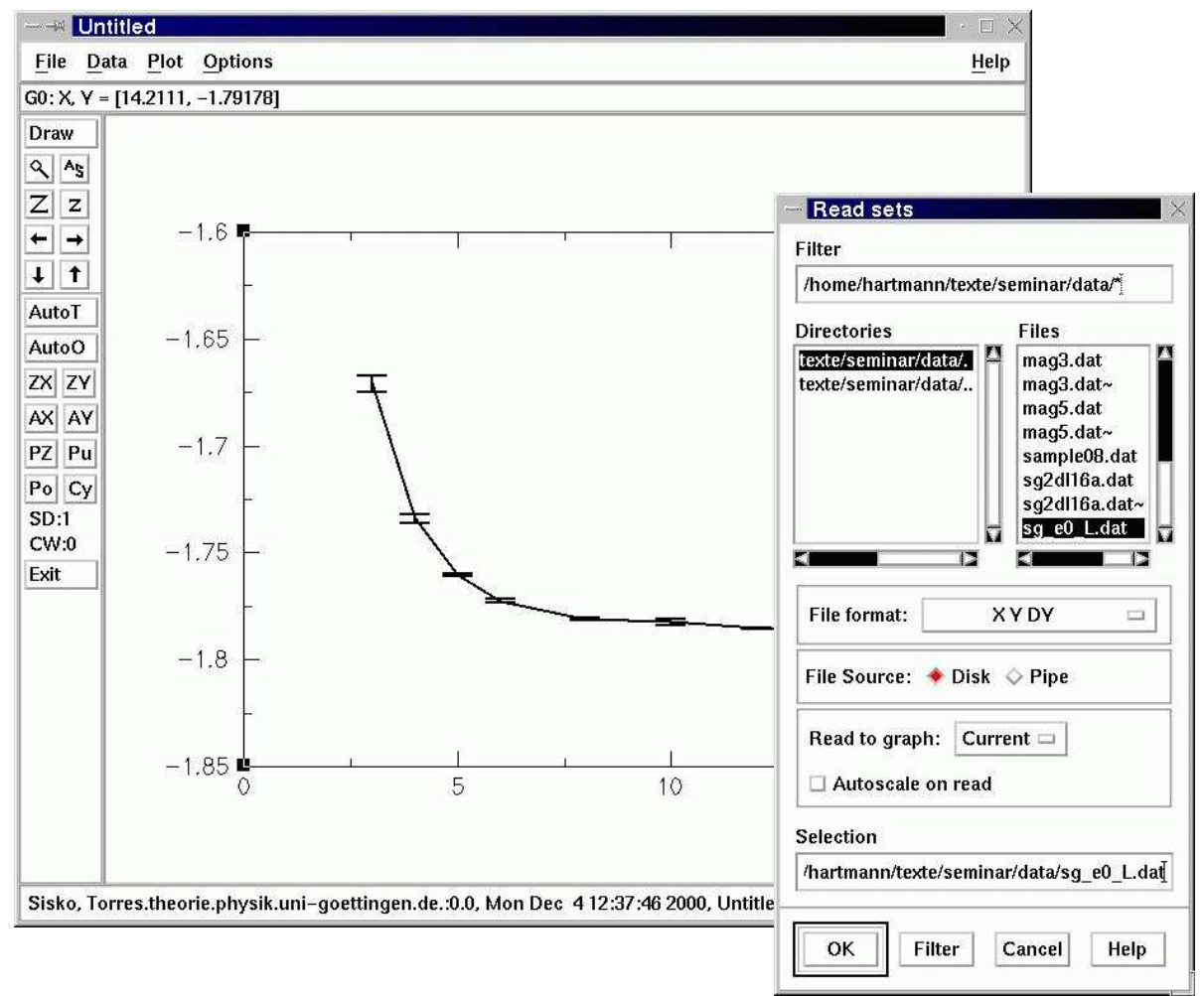

Figure 9: The xmgr program, just after a data file has been loaded, and the AS button has been pressed to adjust the figure range automatically.

mations for the unknown parameters, please note that the exponential operator is denoted by $* *$ and the standard argument for a function definition is $\mathrm{x}$, but this depends only on your choice:

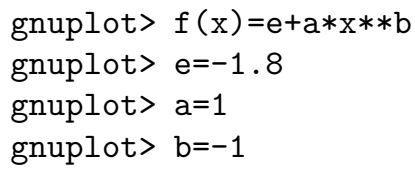

The actual fit is performed via the fit command. The program uses the nonlinear least-squares Marquardt-Levenberg algorithm [3], which allows a fit according to almost all arbitrary functions. To issue the command, you have to state the fit function, the data set and the parameters which are to be adjusted. For our example you enter:

gnuplot> fit $f(x)$ "sg_e0_L.dat" via e,a,b 
Then gnuplot writes log information to the output describing the fitting process. After the fit has converged it prints for the given example:

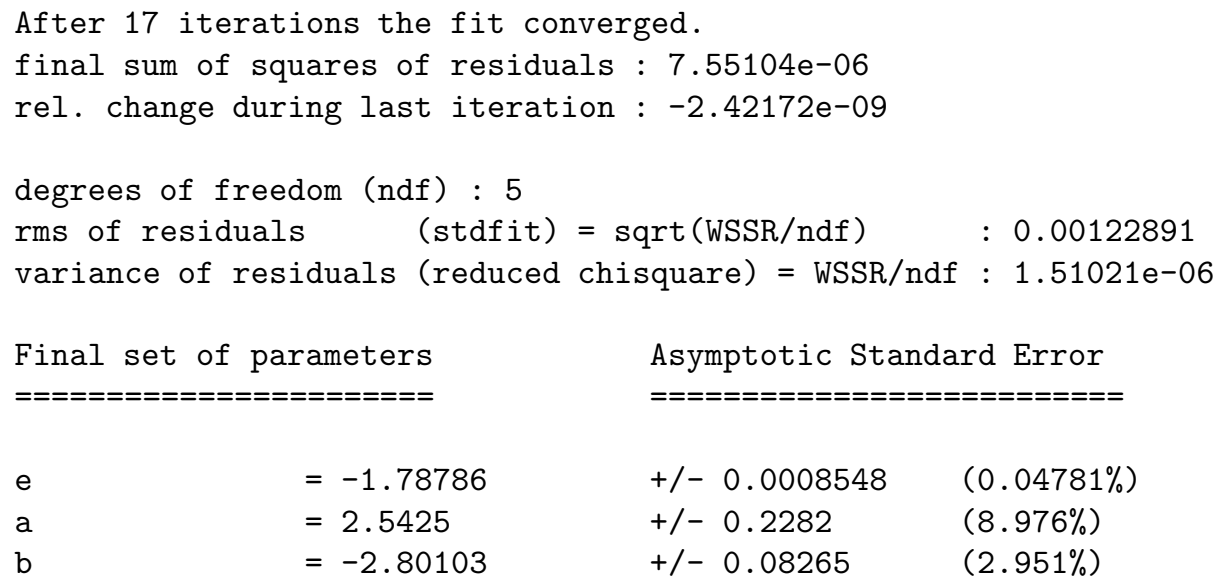

The most interesting lines are those where the results for your parameters along with the standard error are printed. Additionally, the quality of the fit can be estimated by the information provide in the three lines beginning with "degree of freedom". The first of these lines states the number of degrees of freedom, which is just the number of data points minus the number of parameters in the fit. The deviation of the fit function $f(x)$ from the data points $\left(x_{i}, y_{i} \pm \sigma_{i}\right)$ $(i=1, \ldots, N)$ is given by $\chi^{2}=\sum_{i=1}^{N}\left[\frac{y_{i}-f\left(x_{i}\right)}{\sigma_{i}}\right]^{2}$, which is denoted by WSSR in the gnuplot output. A measure of the quality of the fit is the probability $Q$ that the value of $\chi^{2}$ is worse than in the current fit, given the assumption that the datapoints $y_{i}$ are Gaussian distributed with mean $f\left(x_{i}\right)$ and variance one [3]. The larger the value of $Q$, the better is the quality of the fit. To calculate $Q$ you can use the little program $Q$.c 


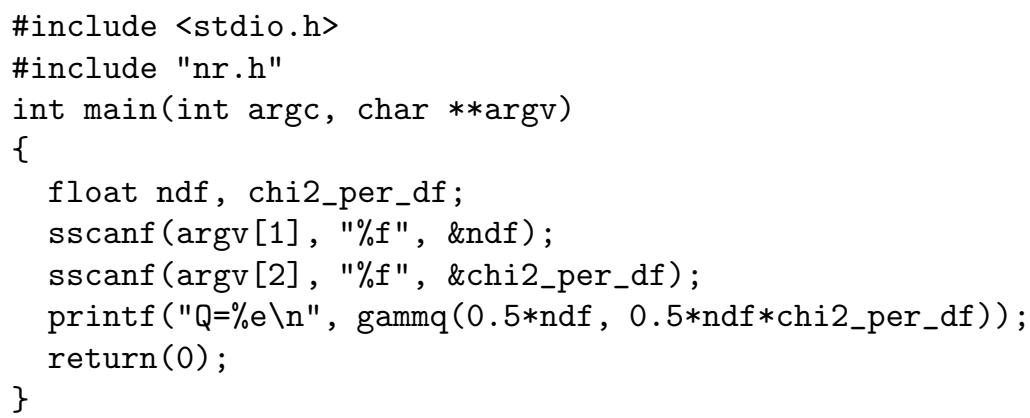

which uses the gammaq function from Numerical Recipes [3]. The program is called in the form $\mathrm{Q}\langle\mathrm{ndf}\rangle\langle\mathrm{WSSR} / \mathrm{ndf}\rangle$, which can be taken from the gnuplot output.

To watch the result of the fit along with the original data, just enter

gnuplot> plot "sg_e0_L.dat" w e, f(x)

The result looks like that shown in Fig. 10

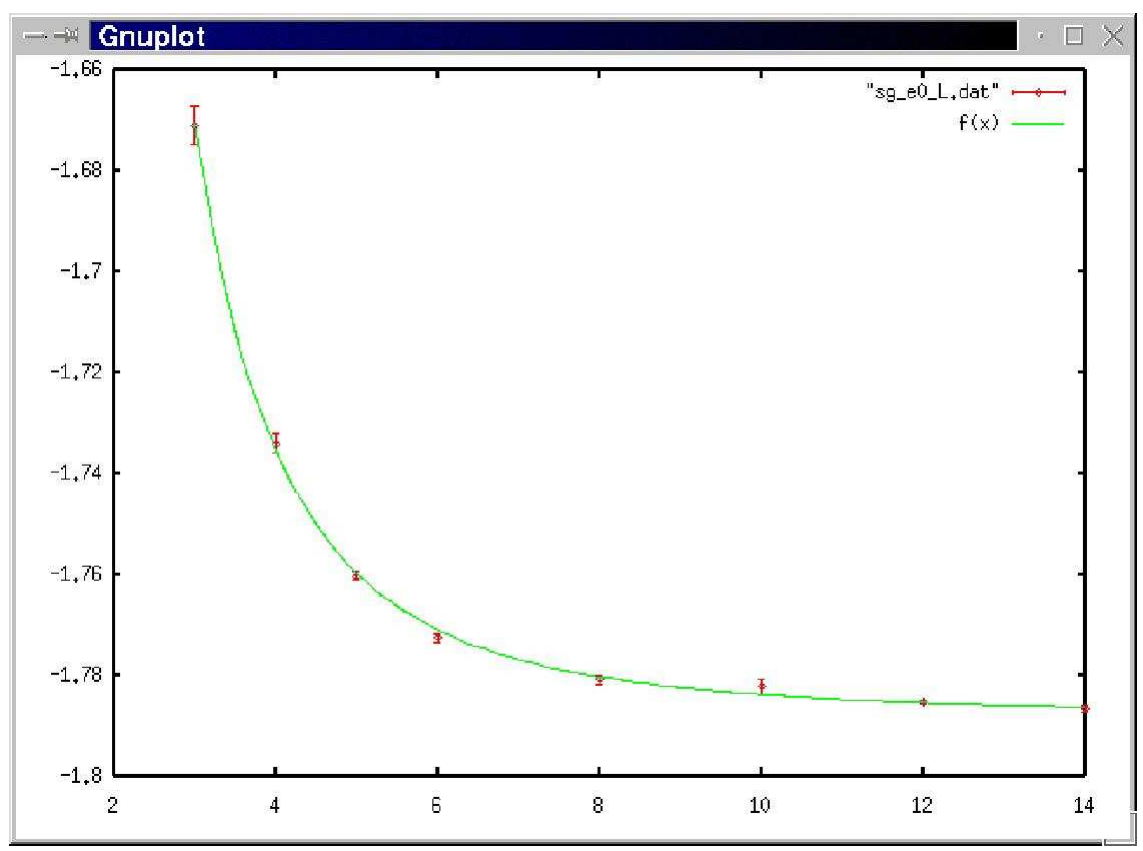

Figure 10: Gnuplot window showing the result of a fit command along with the input data.

Please note that the convergence depends on the initial choice of the parameters. The algorithm may be trapped into a local minimum in case the parameters are 
too far away from the best values. Try the initial values $e=1, a=-3$ and $b=1$ ! Furthermore, not all function parameters have to be subjected to the fitting. Alternatively, you can set some parameters to fixed values and omit them from the list at the end of the fit command. You should also know that in the example given above all data points enter into the result with the same weight. You can tell the algorithm to consider the error bars by typing fit $f(x)$ "sg_e0_L.dat" using 1:2:3 via $\mathrm{a}, \mathrm{b}, \mathrm{c}$. Then, data points with larger error bars have less influence on the results. More on how to use the fit command can be found out when entering help fit.

\subsection{Finite-size Scaling}

Statistical physics describes the behavior of systems with many particles. Usually, realistic system sizes cannot be simulated on current computers. To circumvent this problem, the technique of finite-size scaling has been invented, for an introduction see e.g. Ref. [21]. The basic idea is to simulate systems of different sizes and extrapolate to the large volume limit. Here it is shown how finite-size scaling can be performed with the help of gnuplot [13] or with the special-purpose program fsscale [22]

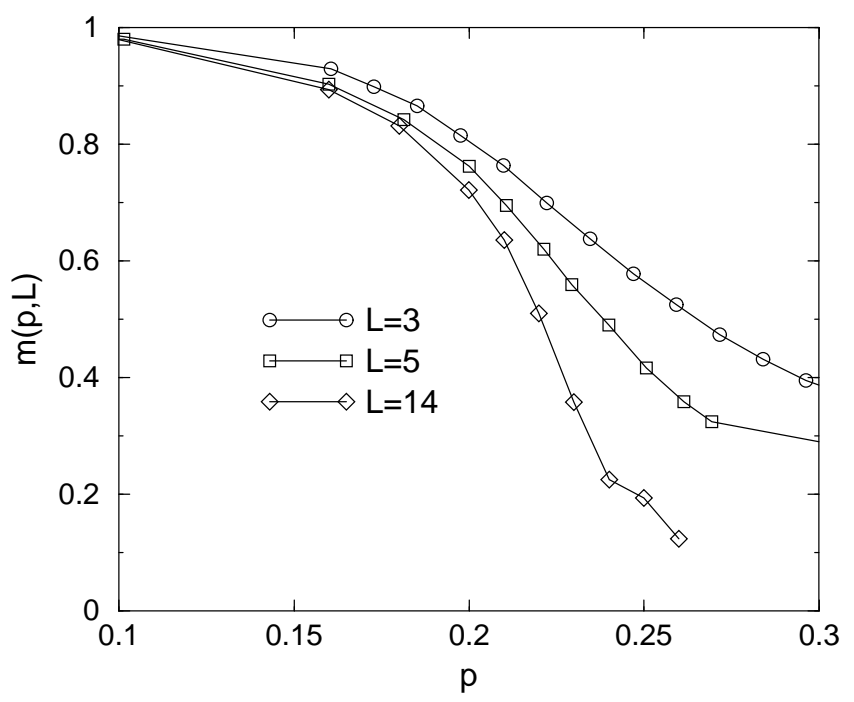

Figure 11: Average ground-state magnetization $m$ of a three-dimensional $\pm J$ spin glass with fractions $p$ of antiferromagnetic bonds. Lines are guides to the eyes only.

As an example, the average ground-state magnetization $m$ of a three-dimensional $\pm J$ spin glass with fractions $p$ of antiferromagnetic and $1-p$ of ferromagnetic bonds is considered. For small values of $p$ the system is expected to have a 
ferromagnetically ordered state. This can be observed in Fig. 11, where the results [23] for different system sizes $L=3,5,14$ are shown.

The critical concentration $p_{\mathrm{c}}$, where the magnetization $m$ vanishes, and the critical behavior of $m$ near the transition are to be obtained. From the theory of finite-size scaling, it is known that the average magnetization $m \equiv\langle M\rangle$ obeys the finite-size scaling form [24]

$$
m(p, L)=L^{-\beta / \nu} \tilde{m}\left(L^{1 / \nu}\left(p-p_{\mathrm{c}}\right)\right)
$$

where $\tilde{m}$ is a universal, i.e. non size-dependent, function. The exponent $\beta$ characterizes the algebraic behavior of the magnetization near $p_{\mathrm{c}}$, while the exponent $\nu$ describes the divergence of the correlation length when $p_{\mathrm{c}}$ is approached. From Eq. (6) you can see that when plotting $L^{\beta / \nu} m(p, L)$ against $L^{1 / \nu}\left(p-p_{\mathrm{c}}\right)$ with correct parameters $\beta, \nu$ the data points for different system sizes should collapse onto a single curve. A good collapse can be obtained by using the values $p_{\mathrm{c}}=0.222, \nu=1.1$ and $\beta=0.27$. The determination of $p_{\mathrm{c}}$ and the exponents can be performed via gnuplot. For that purpose you need a file $\mathrm{m}_{-}$scaling. dat with three columns, where the first column contains the system sizes $L$, the second the values of $p$ and the third contains magnetization $m(p, L)$ for each data point. First, assume that you know the values for $p_{\mathrm{c}}, \nu$ and $\beta$. In this case, the actual plot is done by entering:

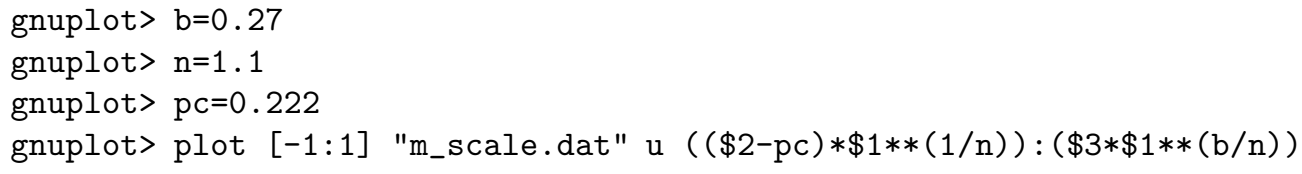

The plot command makes use of the feature that with the $u(\operatorname{sing})$ option you can transform the data of the input in an arbitrary way. For each data set, the variables $\$ 1, \$ 2$ and $\$ 3$ refer to the first, second and third columns, e.g. $\$ 1^{* *}(1 / \mathrm{n})$ raises the system size to the power $1 / \nu$. The resulting plot is shown in Fig. 12. Near the transition $p-p_{\mathrm{c}} \approx 0$ a good collapse of the data points can be observed.

In case you do not know the values of $p_{\mathrm{c}}, \beta, \nu$ you can start with some estimated values, perform the plot, resulting probably in a bad collapse. Then you may alter the parameters iteratively and watch the resulting changes by plotting again. In this way you can converge to a set of parameters, where all data points show a satisfying collapse.

The process of determining the finite-size scaling parameters can be performed more conveniently by using the special purpose program fsscale. It can be obtained free of charge from [22]. This tool allows the scaling parameters to be changed interactively by pressing buttons on the keyboard, making a finitesize scaling fit very convenient to perform. Several different scaling forms are available. To obtain more information, start the program, with fsscale -help. A sample screen-shot is shown in Fig. 13

Please note that the data have to be presented to fsscale in a file containing three columns, where the first column contains the system size, the second the 


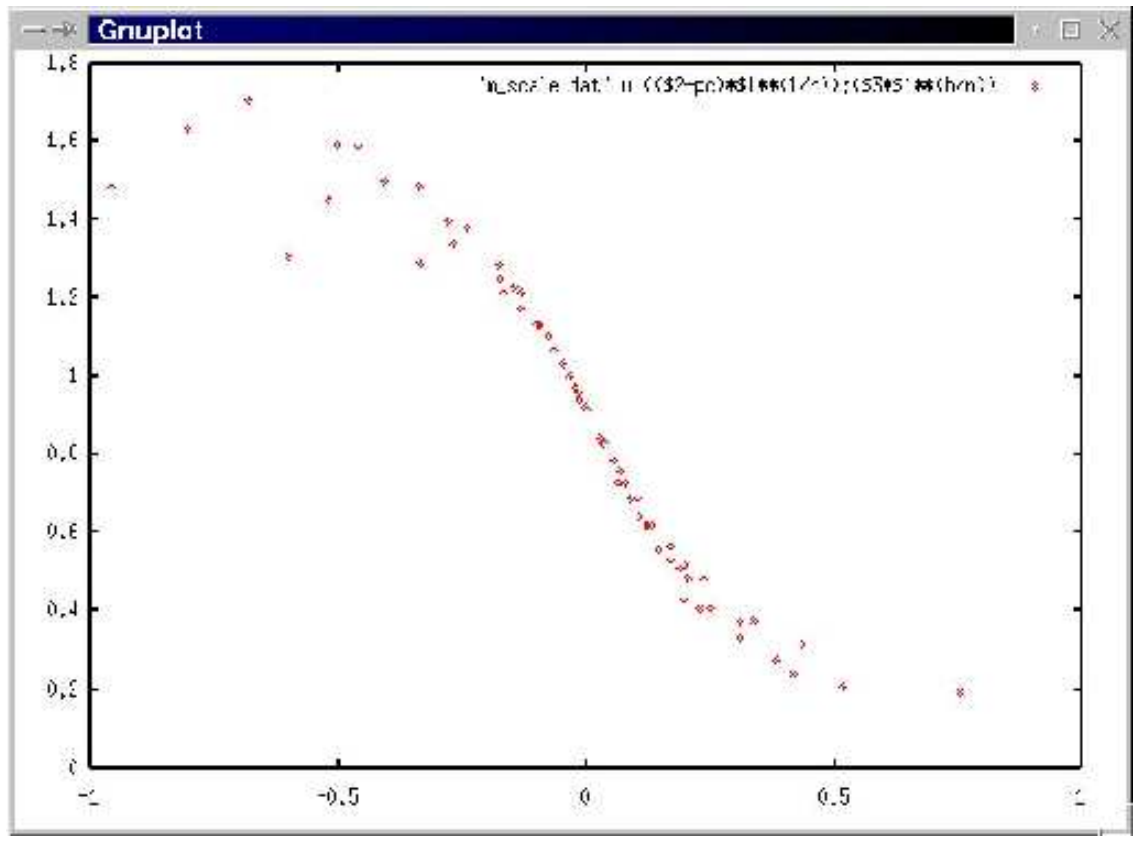

Figure 12: Gnuplot output of a finite-size scaling plot. The ground-state magnetization of a three-dimensional $\pm J$ spin glass as a function of the concentration $p$ of the antiferromagnetic bonds is shown. For the fit, the parameters $p_{\mathrm{c}}=0.222, \beta=0.27$ and $\nu=1.1$ have been used.

$x$-value and the third the $y$-value. If you have only data files with more columns, you can use the standard UNIX tool awk to project out the relevant columns. For example, assume that your data file results. dat has 10 columns, and your are interested in columns 3,8 , and 9 . Then you have to enter:

awk ' \{print $\$ 3, \$ 8, \$ 9\}$ ' results.dat > projected.dat

You can also use awk to perform calulations with the values in the columns, similar to gnuplot, as in

awk '\{print $\$ 1+\$ 2,2.0 * \$ 7, \$ 8 * \$ 1\}$ ' results.dat

\section{Information Retrieval and Publishing}

In this section some basic information regarding searching for literature and preparing your own presentations and publications is given. 


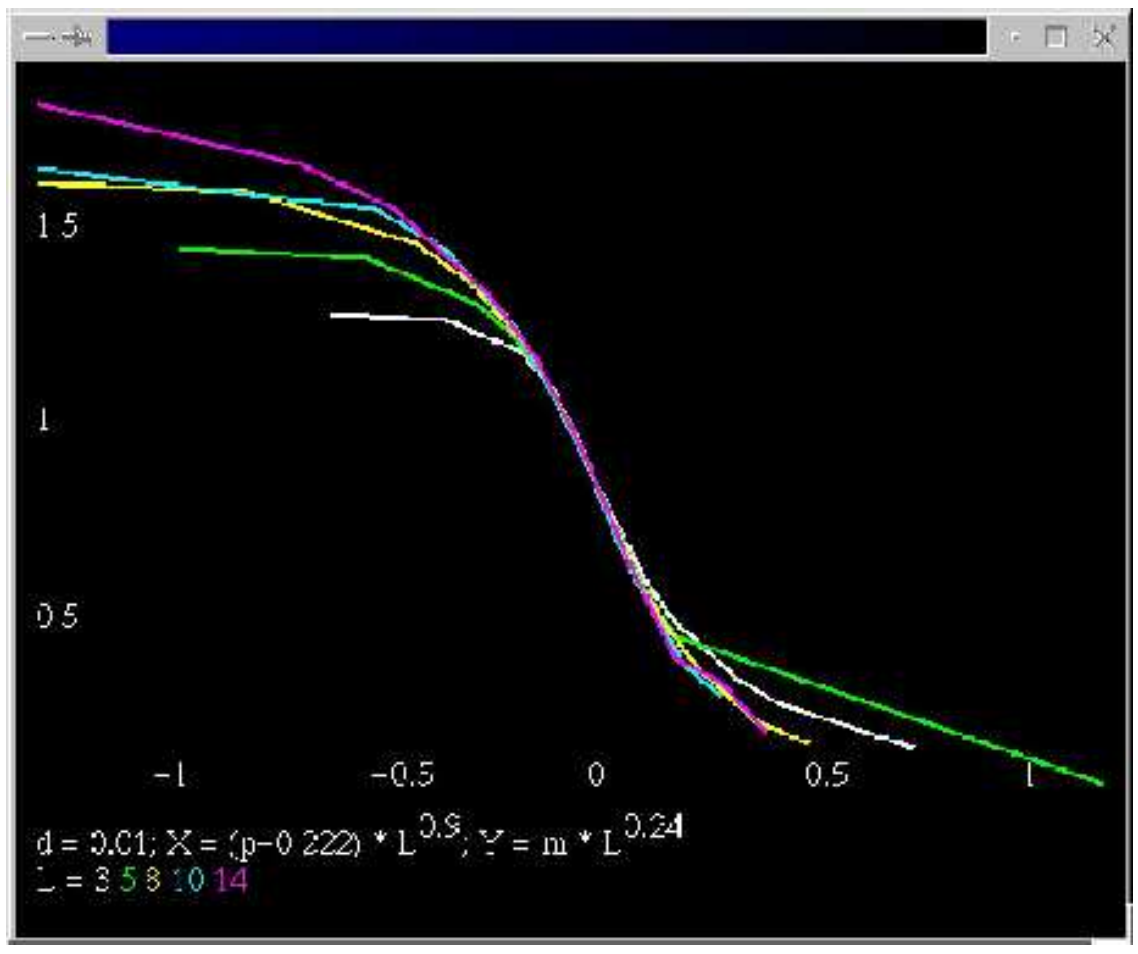

Figure 13: Screen-shot from a window running the fsscale tool.

\subsection{Searching for Literature}

Before contributing to the physical community and even publishing your results, you should be aware of what exists already. This prevents you from redoing something which has been done before by someone else. Furthermore, knowing previous results and many simulation techniques allows you to conduct your own research projects better. Unfortunately, much information cannot be found in textbooks. Thus, you must start to look at the literature. With modern techniques like CD-ROMs and the Internet this can be achieved very quickly. Within this section, it is assumed that you are familiar with the Internet and are able to use a browser. In the following list several sources of information are contained.

- Your local (university) library

Although the amount of literature is limited from space constraints, you should always check your local library for suitable textbooks concerning your area of research. Also many old issues of scientific journals are yet not available through the Internet, thus you may have to copy some articles in the library. 


\section{- Literature databases}

In case you want to obtain all articles from a specific author or all articles on a certain subject, you should consult a literature database. In physics the INSPEC [25] database is the appropriate source of information. Unfortunately, the access is not free of charge. But usually your library should allow access to INSPEC, either via CD-ROMS or via the Internet. If your library/university does not offer an access you should complain.

INSPEC frequently surveys almost all scientific journals in the areas of physics, electronics and computers. For each paper that appears, all bibliographic information along with the abstract are stored. You can search the database for example for author names, keywords (in the abstract or title), publication years or journals. Via INSPEC it is possible to keep track of recent developments happening in a certain field.

There are many other specialized databases. You should consult the web page of your library, to find out to which of them you can access. Modern scientific work is not possible without regularly checking literature databases.

\section{- Preprint server}

In the time of the Internet, speed of publication becomes increasingly important. Meanwhile, many researchers put their publications on the Los Alamos Preprint server [26], where they become available world wide at most 72 (usually 24) hours after submission. The database is free of charge and can be accessed from almost everywhere via a browser. The preprint database is divided into several sections such as astrophysics (astro-ph), condensed matter (cond-mat) or quantum physics (quant-ph). Similar to a conventional literature database, you can search the database, eventually restricted to a section, for author names, publication years or keywords in the title/abstract. But furthermore, after you have found an interesting article, you can download it and print it immediately. File formats are postscript and pdf. The submission can also be in $\mathrm{T}_{\mathrm{E}} \mathrm{X} / \mathrm{ATT}_{\mathrm{EX}}$ (see Sec. 9.2).

Please note that there is no editorial processing at all, that means you do not have any guarantee on the quality of a paper. If you like, you can submit a poem describing the beauty of your garden. Nevertheless, the aim of the server is to make important scientific results available very quickly. Thus, before submitting an article, you should be sure that it is correct and interesting, otherwise you might get a poor reputation.

The preprint server also offers access via email. It is possible to subscribe to a certain subject. Then every working day you will receive a list of all new papers which have been submitted. This is a very convenient way of keeping track of recent developments. But be careful, not everyone submits to the preprint server. Hence, you still have to read scientific journals regularly. 


\section{- Scientific journals}

Journals are the most important resources of information in science. Most of them allow access via the Internet, when your university or institute has subscribed to them. Some of the most important physical journals, which are available online, are published by (in alphabetical order)

- the American Institute of Physics [27]

- the American Physical Society [28]

- Elsevier Science (Netherlands) [29]

- the European Physical Society [30]

- the Institute of Physics (Great Britain) [31]

- Springer Science (Germany) [32]

- Wiley-VCH (USA/Germany) [33]

- World-Scientific (Singapore) [34]

\section{- Citation databases}

In every scientific paper some other articles are cited. Sometimes it is interesting to get the reverse information, i.e. to obtain all papers which are citing a given article $\mathrm{A}$. This can be useful, if one wants to learn about the most recent developments which are triggered by article A. In that case you have to access a citation index. For physics, probably the most important is the Science Citation Index (SCI) which can be accessed via the Web of Science [35]. You have to ask your system administrator or your librarian whether and how you can access it from your site.

The American Physical Society (APS) [28] also includes links to citing articles with the online versions of recent papers. If the citing article is available via the APS as well, you can immediately access the article from the Internet. This works not only for citing papers, but also for cited articles.

\section{- Phys Net}

If you want to have access to the web pages of a certain physics department, you should go via your web browser to the Phys Net pages [36]. They offer a list of all physics departments in the world. Additionally, you will find lists of forthcoming conferences, job offers and many other useful links. Also, the home page of your department probably offers many interesting links to other web pages related to physics.

\section{- Web browsing}

Except for the sources mentioned so far, nowadays much information is available on net. Many researchers present their work, their results and their publications on their home pages. Quite often talks or computer codes can also be downloaded.

In case you cannot find a specific page through the Phys Net (see above), or you are interested in obtaining all web pages concerning a specific subject, 
you should ask a search engine. There are some very popular all purpose engines like Yahoo [37] or Alta Vista [38]. A very convenient way to start a query on several search engines in parallel is a meta search engine, e.g. Metacrawler [39]. To find out more, please contact a search engine.

\subsection{Preparing Publications}

In this section tools for two types of presenting your results are covered: via an article/report or in a talk. For writing papers, it is recommended that you use $T_{E} X / E^{A} T_{E} X$. Data plots can be produced using the programs explained in the last section. For drawing figures and making transparencies, the program xfig offers a large functionality. To create three-dimensional perspective images, the program Povray can be used. $\mathrm{L}_{\mathrm{E}} \mathrm{E} \mathrm{X}, x f i g$ and Povray are introduced in this section.

First, $\mathrm{T}_{\mathrm{E}} \mathrm{X} / \mathrm{L} \mathrm{T} \mathrm{T}_{\mathrm{E}} \mathrm{X}$ is explained. It is a typesetting system rather than a word processor. The basic program is $\mathrm{T}_{\mathrm{E}} \mathrm{X}, \mathrm{IAT}_{\mathrm{E}} \mathrm{X}$ is an extension to facilitate the application. In the area of theoretical computer science, the combination of $\mathrm{T}_{\mathrm{E}} \mathrm{X}$ and $\mathrm{AT}_{\mathrm{E} X} \mathrm{X}$ is a widespread standard. When submitting an article electronically to a scientific journal usually $\mathrm{IAT}_{\mathrm{E} X} \mathrm{X}$ has to be used. Unlike the conventional office packages, with $\mathrm{I}_{\mathrm{E}} \mathrm{X}$ you do not see the text in the form it will be printed, i.e. IATEX is not a WYSIWYG ("What you see is what you get") program. The text is entered in a conventional text editor (like Emacs) and all formatting is done via special commands. An introduction to the $\mathrm{IAT}_{\mathrm{E} X} \mathrm{X}$ language can be found e.g. in Refs. [40, 41]. Although you have to learn several commands, the use of $\mathrm{HT}_{\mathrm{E} X} \mathrm{X}$ has several advantages:

- The quality of the typesetting is excellent. It is much better than selfmade formats. You do not have to care about the layout. But still, you are free to change everything according to your requirements.

- Large projects do not give rise to any problems, in contrast to many commercial office programs. When treating a $\mathrm{AT}_{\mathrm{E}} \mathrm{X}$ text, your computer will never complain when your text is more than 300 pages or contains many huge post-script figures.

- Type setting of formulae is very convenient and fast. You do not have to care about sizes of indices of indices etc. Furthermore, in case you want for example to replace all $\alpha$ in your formulae with $\beta$, this can be done with a conventional replace, by replacing all \alpha strings by a \beta strings. For the case of an office system, please do not ask how to do this conveniently.

- There are many additional packages for enhanced styles such as letters, transparencies or books. The bibtex package is very convenient, which allows a nice literature database to be build up.

- Since you can use a conventional editor, the writing process is very fast. You do not have to wait for a huge packet to come up. 
- On the other hand, if you still prefer a WYSIWYG ("what you see is what you get") system, there is a program called lyx [42] which operates like a conventional word processor but creates $\mathrm{A}_{\mathrm{E}} \mathrm{X}$ files as output. Nevertheless, once you get used to $\mathrm{AT}_{\mathrm{E}} \mathrm{X}$, you will never want to loose it.

Please note that this text was written entirely with $\mathrm{LT}_{\mathrm{E}} \mathrm{X}$. Since $\mathrm{L}_{\mathrm{E}} \mathrm{X}$ is a type setting language, you have to compile your text to create the actual output. Now, an example is given of what a $\mathrm{LT}_{\mathrm{E}} \mathrm{X}$ text looks like and how it can be compiled. This example will just give you an impression of how the system operates. For a complete reference, please consult the literature mentioned above.

The following file example.tex produces a text with different fonts and a formula:

$\backslash$ documentclass [12pt] \{article\}

$\backslash$ begin\{document\}

This is just a small sample text. You can write some words $\{\backslash$ em emphasized $\} \backslash$, or in $\{\backslash$ bf bold face $\}$. Also different $\{\backslash$ small sizes $\}$ are possible.

An empty line generates a new paragraph. \LaTeX\ is very convenient for writing formulae, e.g.

The first line introduces the type of the text (article, which is the standard) and the font size. You should note that all tex commands begin with a backslash $(\backslash)$, in case you want to write a backslash in your text, you have to enter $\$ \backslash$ backslash\$. The actual text is written between the lines starting with $\backslash$ begin $\{$ document $\}$ and ending with $\backslash$ end $\{$ document $\}$. You can observe some commands such as $\backslash$ em, $\backslash$ bf or $\backslash$ small. The \{\} braces are used to mark blocks of text. Mathematical formulae can be written e.g. with \begin\{equation\} } and $\backslash$ end $\{$ equation\}. For the mathematical mode a huge number of commands exists. Here only examples for Greek letters ( $\backslash$ alpha), subscripts ( $\left.x_{-} i\right)$, fractions $(\backslash$ frac), integrals ( $\backslash$ int $)$ and vectors $(\backslash$ vec $)$ are given.

The text can be compiled by entering latex example.tex. This is the command for UNIX, but $\mathrm{LT}_{\mathrm{E}} \mathrm{X}$ exists for all operating systems. Please consult the documentation of your local installation.

The output of the compiling process is the file example.dvi, where "dvi" means "device independent". The .dvi file can be inspected on screen by a viewer via entering xdvi example.dvi or converted into a postscript file via typing dvips -o example.ps example.dvi and then transferred to a printer. On many systems it can be printed directly as well. The result will look like this:

This is just a small sample text. You can write some words emphasized, or in bold face. Also different sizes are possible. 
An empty line generates a new paragraph. $\mathrm{LT}_{\mathrm{E}} \mathrm{X}$ is very convenient for writing formulae, e.g.

$$
M_{i}(t)=\frac{1}{L^{3}} \int_{V} x_{i} \rho(\vec{x}, t) d^{3} \vec{x}
$$

This example should be sufficient to give you an impression of what the philosophy of $\mathrm{AHT}_{\mathrm{E} X}$ is. Comprehensive instructions are beyond the scope of this section, please consult the literature [40,41].

Under UNIX/Linux, the spell checker ispell is available. It allows a simple spell check to be performed. The tool is built on a dictionary, i.e. a huge list of known words. The program scans any given text, also a special $\mathrm{LT}_{\mathrm{E}} \mathrm{X}$ mode is available. Every time a word occurs, which is not contained in the list, ispell stops. Should similar words exist in the list, they are suggested. Now the user has to decide whether the word should be replaced, changed, accepted or even added to the dictionary. The whole text is treated in this way. Please note that many mistakes cannot be found in this way, especially when the misspelled word is equal to another word in the dictionary. However, at least ispell finds many spelling mistakes quickly and conveniently, so you should use the tool. Most scientific texts do not only contain text, formulae and curves, but also schematic figures showing the models, algorithms or devices covered in the publication. A very convenient but also simple tool to create such figures is $x f i g$. It is a window based vector-oriented drawing program. Among its features are the creation of simple objects like lines, arrows, polylines, splines, arcs as well as rectangles, circles and other closed, possibly filled, areas. Furthermore you can create text or include arbitrary (eps, jpg) pictures files. You may place the objects on different layers which allows complex sceneries to be created. Different simple objects can be combined into more complex objects. For editing you can move, copy, delete, rotate or scale objects. To give you an impression what $x f i g$ looks like, in Fig. 14 a screen-shot is shown, displaying $x f i g$ with the picture that is shown in Fig. 1. Again, for further help, please consult the online help function or the man pages.

The figures can be saved in the internal fig format, and exported in several file formats such as (encapsulated) postscript, $\mathrm{AT}_{\mathrm{E} X}$, Jpeg, Tiff or bitmap. The $x$ fig program can be called in a way that it produces just an output file with a given fig input file. This is very convenient when you have larger projects where some small picture objects are contained in other pictures and you want to change the appearance of the small objects in all other files. With the help of the make program pretty large projects can be realized.

Also, $x$ fig is very convenient when creating transparencies for talks, which is the standard method of presenting results in physics. With different colors, text sizes and all the objects mentioned before, very clear transparencies can be created quickly. The possibility of including picture files, like postscript files which were created by a data plotting program such as $x m g r$, is very helpful. In the beginning it may seem that more effort is necessary than when creating the transparencies by hand. However, once you have a solid base of transparencies you can reuse many parts and preparing a talk may become a question of 


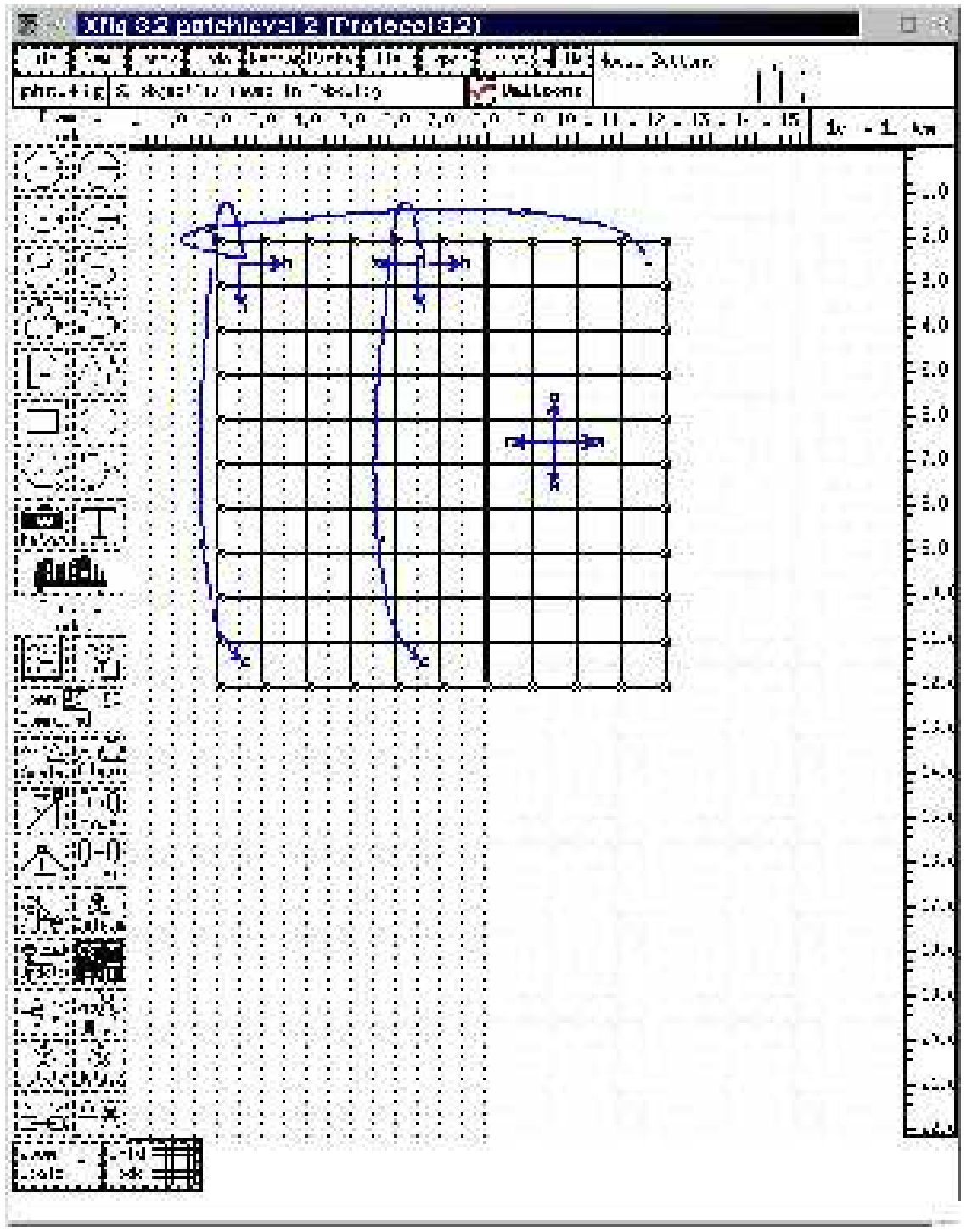

Figure 14: A sample screen-shot showing the $x f i g$ program.

minutes. In particular, when your handwriting looks awful, the audience will be much obliged for transparencies prepared with $x f i g$.

Last but not least, please note that $x$ fig is vector oriented, but not pixel oriented. Therefore, you cannot treat pictures like jpg files (e.g. photos) and apply operations like smoothing, sharpening or filtering. For these purposes the package gimp is suitable. It is freely available again from GNU [5]. 
It is also possible to draw three-dimensional figures with $x f i g$, but there is no special support for it. This means, xfig has only a two-dimensional coordinate system. A very convenient and powerful tool for making three-dimensional figures is Povray (Persistence Of Vision RAYtraycer). Here, again, only a short example is given, for a detailed documentation please refer to the home page [43], where the program can be downloaded for many operating systems free of charge.

Povray is, as can be realized from its name, a raytracer. This means you present a scene consisting of several objects to the program. These objects have characteristics like color, reflectivity or transparency. Furthermore the position of one or several light sources and a virtual camera have to be defined. The output of a raytracer is a photo-realistic picture of the scene, seen through the camera. The name "raytracer" originates from the fact that the program creates a picture by starting several rays of light at the light sources and traces their way through the scene, where they may be absorbed, reflected or refracted, until they hit the camera, disappear into infinity or become too weak. Hence, the creation of a picture may take a while, depending on the complexity of the scene.

A scene is described in a human readable file, it can be entered with any text editor. But for more complex scenes, special editors exist, which allow a scene to be created interactively. Also several tools for making animations are available on the Internet. Here, a simple example is given. The scene consists of three spheres connected by two cylinders, forming a molecule. Furthermore, a light source, a camera, an infinite plane and the background color are defined. Please note that a sphere is defined by its center and a radius and a cylinder by two end points and a radius. Additionally, for all objects color information has to be included, the center sphere is slightly transparent. The scene description file test 1 . pov reads as follows:

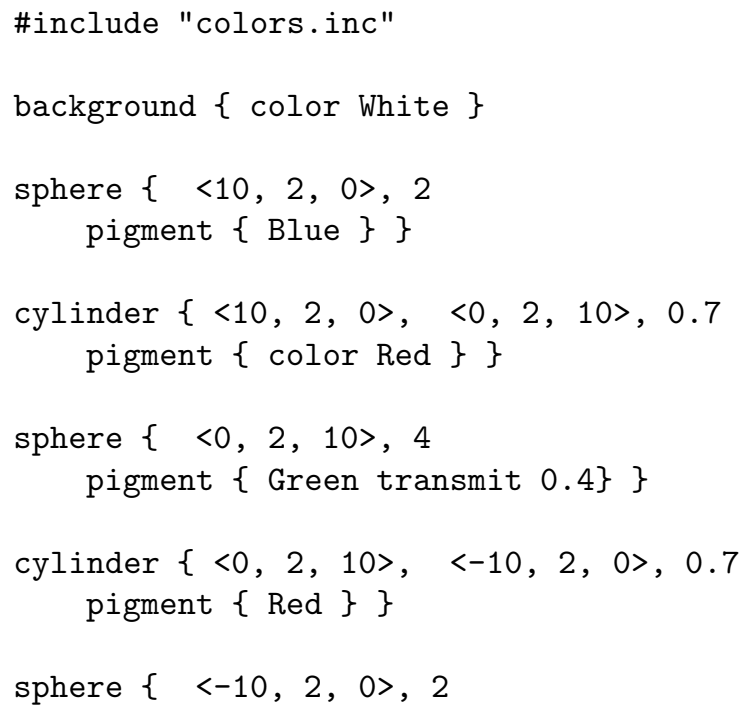




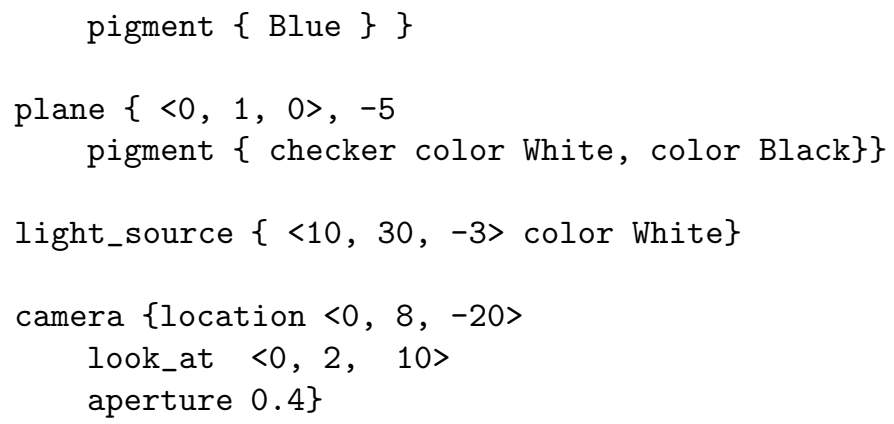

The creation of the picture is started by calling (here on a Linux system via command line) $\mathrm{x}$-povray $+\mathrm{I}$ test 1 .pov. The resulting picture is shown in Fig. 15 , please note the shadows on the plane.

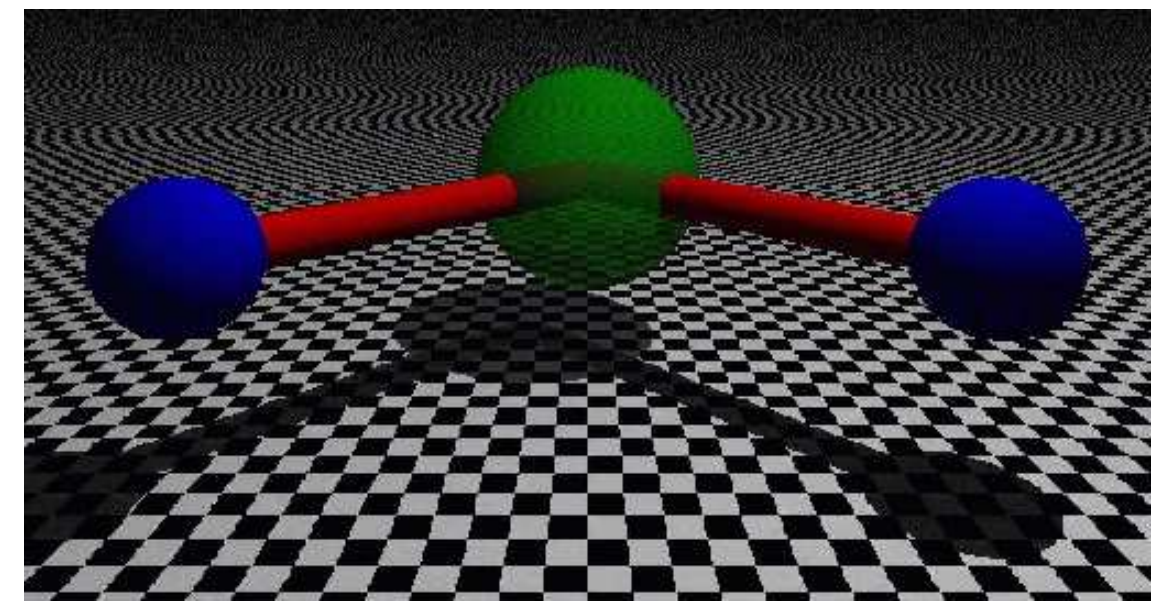

Figure 15: A sample scene created with Povray.

Povray is really powerful. You can create almost arbitrarily shaped objects, combine them into complex objects and impose many transformations. Also special effects like blurring or fog are available. All features of Povray are described in a 400 page manual. The use of Povray is widespread in the artists community. For scientists it is very convenient as well, because you can easily convert e.g. configuration files of molecules or three-dimensional domains of magnetic systems into nice looking perspective pictures. This can be accomplished by writing a small program which reads e.g your configuration file containing a list of positions of atoms and a list of links, and puts for every atom a sphere and for every link a cylinder into a Povray scene file. Finally the program must add suitable chosen light sources and a camera. Then, a three-dimensional pictures is created by calling Povray.

The tools described in this section, should allow all technical problems occurring 
in the process of preparing a publication (a "paper") to be solved. Once you have prepared it, you should give it to at least one other person, who should read it carefully. Probably he/she will find some errors or indicate passages which might be difficult to understand or that are misleading. You should always take such comments very seriously, because the average reader knows much less about your problem than you do.

After all necessary changes have been performed, and you and other readers are satisfied with the publication, you can submit it to a scientific journal. You should choose a journal which suits your paper. Where to submit, you should discuss with experienced researchers. It is not possible to give general advice on this issue. Nevertheless, technically the submission can be performed nowadays almost everywhere electronically. For a list of publishers of some important journals in physics, please see Sec. 9.1. Submitting one paper to several journals in parallel is not allowed. However, you should also consider submitting to the preprint server [25] as well to make your results quickly available to the physics community.

Nevertheless, although this text provides many useful hints concerning performing computer simulations, the main part of the work is still having good ideas and carefully conducting the actual research projects.

\section{References}

[1] I. Sommerville, Software Engineering, (Addisin-Wesley, Reading (MA) 1989)

[2] C. Ghezzi, M. Jazayeri, and D. Mandrioli, Fundamentals of Software Engineering, (Prentice Hall, London 1991)

[3] W.H. Press, S.A. Teukolsky, W.T. Vetterling, and B.P. Flannery, Numerical Recipes in $C$ (Cambridge University Press, Cambridge 1995)

[4] K. Mehlhorn and St. Näher, The LEDA Platform of Combinatorial and Geometric Computing (Cambridge University Press, Cambridge 1999);

see also http://www.mpi-sb.mpg.de/LEDA/leda.html

[5] M. Loukides and A. Oram, Programming with GNU Software, (O'Reilly, London 1996);

see also http://www.gnu.org/manual

[6] H.R. Lewis and C.H. Papadimitriou, Elements of the Theory of Computation, (Prentice Hall, London 1981)

[7] J. Rumbaugh, M. Blaha, W. Premerlani, F. Eddy, and W. Lorensen, ObjectOriented Modeling and Design, (Prentice Hall, London 1991)

[8] R. Johnsonbaugh and M. Kalin, Object Oriented Programming in $\mathrm{C}++$, (Macmillan, London 1994) 
[9] J. Skansholm, $C++$ from the Beginning, (Addisin-Wesley, Reading (MA) 1997)

[10] Mail to hartmann@theorie.physik.uni-goettingen.de

[11] B.W. Kernighan and D.M. Ritchie, The C Programming Language, (Prentice Hall, London 1988)

[12] A. Oram and S. Talbott, Managing Projects With Make, (O'Reilly, London 1991)

[13] The programs and manuals can be found on http://www.gnu.org. For some there is a texinfo file. To read it, call the editor 'emacs' and type $<\mathrm{crtl}>+$ 'h' and then ' $\mathrm{i}$ ' to start the texinfo mode.

[14] J. Phillips, The Nag Library: A Beginner's Guide (Oxford University Press, Oxford 1987);

see also http://www.nag.com

[15] A. Heck, Introduction to Maple, (Springer-Verlag, New York 1996)

[16] B.J.T. Morgan, Elements of Simulation, (Cambridge University Press, Cambridge 1984)

[17] A.M. Ferrenberg, D.P. Landau and Y.J. Wong, Phys. Rev. Lett. 69, 3382 (1992); I. Vattulainen, T. Ala-Nissila and K. Kankaala, Phys. Rev. Lett. 73, 2513 (1994)

[18] J.F. Fernandez and C. Criado, Phys. Rev. E 60, 3361 (1999)

[19] http://www.cs.colorado.edu/homes/zorn/public_html/MallocDebug.html

[20] The tool can be obtained under the gnu public license from http://www.gnu.org/software/checker/checker.html

[21] J. Cardy, Scaling and Renormalization in Statistical Physics, (Cambridge University Press, Cambridge 1996)

[22] The program fsscale is written by A. Hucht, please contact him via email: fred@thp.Uni-Duisburg.DE

[23] A.K. Hartmann, Phys. Rev. B 59 , 3617 (1999)

[24] K. Binder and D.W. Heermann, Monte Carlo Simulations in Statistical Physics, (Springer, Heidelberg 1988)

[25] http://www.inspec.org/publish/inspec/

[26] http://xxx.lanl.gov/

[27] http://www.aip.org/ojs/service.html 
[28] http://publish.aps.org/

[29] http://www.elsevier.nl

[30] http://www.eps.org/publications.html

[31] http://www.iop.org/Journals/

[32] http://www.springer.de/

[33] http://www.wiley-vch.de/journals/index.html

[34] http://ejournals.wspc.com.sg/journals.html

[35] http://wos.isiglobalnet.com/

[36] http://physnet.uni-oldenburg.de/PhysNet/physnet.html

[37] http://www.yahoo.com/

[38] http://www.altavista.com/

[39] http://www.metacrawler.com/index.html

[40] L. Lamport and D. Bibby, LaTeX : A Documentation Preparation System User's Guide and Reference Manual, (Addison Wesley, Reading (MA) 1994)

[41] http://www.tug.org/

[42] http://www.lyx.org/

[43] http://www.povray.org/ 